\title{
ARITHMETIC INTERSECTION ON GSPIN RAPOPORT-ZINK SPACES
}

\author{
CHAO LI AND YIHANG ZHU
}

\begin{abstract}
We prove an explicit formula for the arithmetic intersection number of diagonal cycles on GSpin Rapoport-Zink spaces in the minuscule case. This is a local problem arising from the arithmetic Gan-GrossPrasad conjecture for orthogonal Shimura varieties. Our formula can be viewed as an orthogonal counterpart of the arithmetic-geometric side of the arithmetic fundamental lemma proved by Rapoport-Terstiege-Zhang in the minuscule case.
\end{abstract}

\section{CONTENTS}

1. Introduction

2. GSpin Rapoport-Zink spaces

3. The intersection problem and the point-counting formula

4. The reducedness of minuscule special cycles

5. The intersection length formula

References

\section{INTRODUCTION}

1.1. Motivation. The arithmetic Gan-Gross-Prasad conjectures (arithmetic GGP) generalize the celebrated Gross-Zagier formula to higher dimensional Shimura varieties ([GGP12, §27], [Zha12, §3.2]). It is a conjectural identity relating the heights of certain algebraic cycles on Shimura varieties to the central derivative of certain Rankin-Selberg $L$-functions. Let us briefly recall the rough statement of the conjecture. The diagonal embeddings of unitary groups

$$
H=\mathrm{U}(1, n-1) \hookrightarrow G=\mathrm{U}(1, n-1) \times \mathrm{U}(1, n)
$$

or of orthogonal groups

$$
H=\mathrm{SO}(2, n-1) \hookrightarrow G=\mathrm{SO}(2, n-1) \times \mathrm{SO}(2, n),
$$

induces an embedding of Shimura varieties $\mathrm{Sh}_{H} \hookrightarrow \mathrm{Sh}_{G}$. We denote its image by $\Delta$ and call it the diagonal cycle or the GGP cycle on $\mathrm{Sh}_{G}$. Let $\pi$ be a tempered cuspidal automorphic representation on $G$ appearing in the middle cohomology of $\mathrm{Sh}_{G}$. Let $\Delta_{\pi}$ be the (cohomological trivialization) of the $\pi$-component of $\Delta$. The arithmetic GGP conjecture asserts that the (conditional) Beilinson-Bloch-Gillet-Soulé height of $\Delta_{\pi}$ should be given by the central derivative of a certain Rankin-Selberg $L$-function $L(s, \pi)$ up to simpler factors

$$
\left\langle\Delta_{\pi}, \Delta_{\pi}\right\rangle \sim L^{\prime}(1 / 2, \pi) .
$$

Date: February 28, 2017.

2010 Mathematics Subject Classification. 11G18, 14G17; secondary 22E55.

Key words and phrases. Arithmetic Gan-Gross-Prasad conjecture, Rapoport-Zink spaces, spinor groups, special cycles. 
The Gross-Zagier formula [GZ86] and the work of Gross, Kudla, Schoen (GK92, GS95]) can be viewed as the special cases $n=1$ and $n=2$ in the orthogonal case correspondingly. The recent work of Yuan-ZhangZhang ([YZZ13, [YZZ]) has proved this conjecture for $n=1,2$ in the orthogonal case in vast generality.

In the unitary case, $\mathrm{W}$. Zhang has proposed an approach for general $n$ using the relative trace formula of Jacquet-Rallis. The relevant arithmetic fundamental lemma relates an arithmetic intersection number of GGP cycles on unitary Rapoport-Zink spaces with a derivative of orbital integrals on general linear groups. The arithmetic fundamental lemma has been verified for $n=1,2$ ([Zha12] ) and for general $n$ in the minuscule case by Rapoport-Terstiege-Zhang RTZ13.

In the orthogonal case, very little is known currently beyond $n=1,2$ and no relative trace formula approach has been proposed yet. However it is notable that R. Krishna [Kri16] has recently established a relative trace formula for the case $\mathrm{SO}(2) \times \mathrm{SO}(3)$ and one can hope that his method will generalize to formulate a relative trace formula approach for general $\mathrm{SO}(n-1) \times \mathrm{SO}(n)$.

Our goal in this article is to establish an orthogonal counterpart of the arithmetic-geometric side of the arithmetic fundamental lemma in RTZ13, namely to formulate and compute the arithmetic intersection of GGP cycles on GSpin Rapoport-Zink spaces in the minuscule case.

1.2. The main results. Let $p$ be an odd prime. Let $k=\overline{\mathbb{F}}_{p}, W=W(k), K=W[1 / p]$ and $\sigma \in \operatorname{Aut}(W)$ be the lift of the absolute $p$-Frobenius on $k$. Let $n \geq 4$. Let $V^{b}$ be a self-dual quadratic space over $\mathbb{Z}_{p}$ of rank $n-1$ and let $V=V^{\mathrm{b}} \oplus \mathbb{Z}_{p} x_{n}$ (orthogonal direct sum) be a self-dual quadratic space over $\mathbb{Z}_{p}$ of rank $n$, where $x_{n}$ has norm 1. Associated to the embedding of quadratic spaces $V^{b} \hookrightarrow V$ we have an embedding of algebraic groups $G^{b}=\operatorname{GSpin}\left(V^{b}\right) \hookrightarrow G=\mathrm{GSpin}(V)$ over $\mathbb{Z}_{p}$. After suitably choosing compatible local unramified Shimura-Hodge data $\left(G^{b}, b^{b}, \mu^{b}, C\left(V^{b}\right)\right) \hookrightarrow(G, b, \mu, C(V))$, we obtain a closed immersion of the associated GSpin Rapoport-Zink spaces

$$
\delta: \mathrm{RZ}^{\mathrm{b}} \hookrightarrow \mathrm{RZ} .
$$

See $\$ 2$ for precise definitions and see $\$ 3.2$ for the moduli interpretation of $\delta$. The space RZ is an example of Rapoport-Zink spaces of Hodge type, recently constructed by Kim Kim13] and Howard-Pappas HP15. It is a formal scheme over $\operatorname{Spf} W$, parameterizing deformations of a $p$-divisible group $\mathbb{X}_{0} / k$ with certain crystalline Tate tensors (coming from the defining tensors of $G$ inside some $\mathrm{GL}_{N}$ ). Roughly speaking, if $X^{\mathrm{b}}$ is the $p$-divisible group underlying a point $x \in \mathrm{RZ}^{\mathrm{b}}$, then the $p$-divisible group underlying $\delta(x) \in \mathrm{RZ}$ is given by $X=X^{b} \oplus X^{b}$.

Remark 1.2.1. The datum $(G, b, \mu, C(V))$ is chosen such that the space RZ provides a $p$-adic uniformization

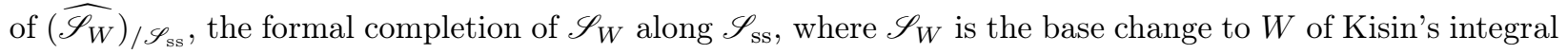
model ([Kis10]) of a GSpin Shimura variety (which is of Hodge type) at a good prime $p$, and $\mathscr{S}_{\text {ss }}$ is the supersingular locus (= the basic locus in this case) of the special fiber of $\mathscr{S}_{W}$ (see [HP15, 7.2]).

The group $J_{b}\left(\mathbb{Q}_{p}\right)=\{g \in G(K): g b=b \sigma(g)\}$ is the $\mathbb{Q}_{p}$-points of an inner form of $G$ and acts on RZ via its action on the fixed $p$-divisible group $\mathbb{X}_{0}$. Let $g \in J_{b}\left(\mathbb{Q}_{p}\right)$. As explained in 3 , the intersection of the GGP cycle $\Delta$ on $\mathrm{RZ}^{\mathrm{b}} \times_{W} \mathrm{RZ}$ and its $g$-translate leads to study of the formal scheme

$$
\delta\left(\mathrm{RZ}^{\mathrm{b}}\right) \cap \mathrm{RZ}^{g},
$$

where $\mathrm{RZ}^{g}$ denotes the $g$-fixed points of RZ.

We call $g \in J_{b}\left(\mathbb{Q}_{p}\right)$ regular semisimple if

$$
L(g):=\mathbb{Z}_{p} x_{n}+\mathbb{Z}_{p} g x_{n}+\cdots+\mathbb{Z}_{p} g^{n-1} x_{n}
$$


is a free $\mathbb{Z}_{p}$-module of rank $n$. Let $L(g)^{\vee}$ denote the dual lattice of $L(g)$. We further call $g$ minuscule if $L(g) \subset L(g)^{\vee}$ (i.e. the quadratic form restricted to $L(g)$ is valued in $\mathbb{Z}_{p}$ ), and $L(g)^{\vee} / L(g)$ is a $\mathbb{F}_{p^{\text {-vector }}}$ space. See Definition 3.3 .2 for equivalent definitions. When $g \in J_{b}\left(\mathbb{Q}_{p}\right)$ is regular semisimple and minuscule, we will show that the formal scheme (1.2.1.1) is in fact a 0 -dimensional scheme of characteristic $p$. Our main theorem is an explicit formula for its arithmetic intersection number (i.e., the total $W$-length of its local rings).

To state the formula, assume $g$ is regular semisimple and minuscule, and suppose $\mathrm{RZ}^{g}$ is nonempty. Then $g$ stabilizes both $L(g)^{\vee}$ and $L(g)$ and thus acts on the $\mathbb{F}_{p}$-vector space $L(g)^{\vee} / L(g)$. Let $P(T) \in \mathbb{F}_{p}[T]$ be the characteristic polynomial of $g$ acting on $L(g)^{\vee} / L(g)$. For any irreducible polynomial $R(T) \in \mathbb{F}_{p}[T]$, we denote its multiplicity in $P(T)$ by $m(R(T)$ ). Moreover, for any polynomial $R(T)$, we define its reciprocal by

$$
R^{*}(T):=T^{\operatorname{deg} R(T)} \cdot R(1 / T) .
$$

We say $R(T)$ is self-reciprocal if $R(T)=R^{*}(T)$. Now we are ready to state our main theorem:

Theorem A. Let $g \in J_{b}\left(\mathbb{Q}_{p}\right)$ be regular semisimple and minuscule. Assume $\mathrm{RZ}^{g}$ is non-empty. Then

(1) (Corollary 5.1.2) $\delta\left(\mathrm{RZ}^{\mathrm{b}}\right) \cap \mathrm{RZ}^{g}$ is a scheme of characteristic $p$.

(2) (Theorem 3.6.4) $\delta\left(\mathrm{RZ}^{\mathrm{b}}\right) \cap \mathrm{RZ}^{g}$ is non-empty if and only if $P(T)$ has a unique self-reciprocal monic irreducible factor $Q(T) \mid P(T)$ such that $m(Q(T))$ is odd. In this case, $p^{\mathbb{Z}} \backslash\left(\delta\left(\mathrm{RZ}^{b}\right) \cap \mathrm{RZ}{ }^{g}\right)(k)$ is finite and has cardinality

$$
\operatorname{deg} Q(T) \cdot \prod_{R(T)}(1+m(R(T)))
$$

where $R(T)$ runs over all non-self-reciprocal monic irreducible factors of $P(T)$. Here, the group $p^{\mathbb{Z}}$ acts on $\mathrm{RZ}$ via the central embedding $p^{\mathbb{Z}} \hookrightarrow J_{b}\left(\mathbb{Q}_{p}\right)$, and the action stabilizes $\delta\left(\mathrm{RZ}^{\mathrm{b}}\right) \cap \mathrm{RZ}^{g}$.

(3) (Corollary 5.4.2) Let $c=\frac{m(Q(T))+1}{2}$. Then $1 \leq c \leq n / 2$. Assume $p>c$. Then $\delta\left(\mathrm{RZ}^{b}\right) \cap \mathrm{RZ}^{g}$ is a disjoint union over its $k$-points of copies of Spec $k[X] / X^{c}$. In particular, the intersection multiplicity at each $k$-point of $\delta\left(\mathrm{RZ}^{b}\right) \cap \mathrm{RZ}^{g}$ is the same and equals $c$.

Along the way we also prove a result that should be of independent interest. In [HP15, Howard-Pappas define closed formal subschemes $\mathrm{RZ}_{\Lambda}$ of $\mathrm{RZ}$ for each vertex lattice $\Lambda$ (recalled in 2 ). Howard-Pappas study the reduced subscheme $\mathrm{RZ}_{\Lambda}^{\text {red }}$ detailedly and prove that they form a nice stratification of $\mathrm{RZ}^{\text {red }}$. We prove:

Theorem B (Theorem 4.2.11). $\mathrm{RZ}_{\Lambda}=\mathrm{RZ}_{\Lambda}^{\mathrm{red}}$ for each vertex lattice $\Lambda$.

1.3. Novelty of the method. The results Theorem A and Theorem B are parallel to the results in RTZ13] for unitary Rapoport-Zink spaces. The main new difficulty in the GSpin case is due to the fact that, unlike the unitary case, the GSpin Rapoport-Zink spaces are not of PEL type. They are only of Hodge type, and as for now they lack full moduli interpretations that are easy to work with directly (see Remark 2.4.1).

In [RTZ13, the most difficult parts are the reducedness of minuscule special cycles [RTZ13, Theorem 10.1] and the intersection length formula [RTZ13, Theorem 9.5]. They are the analogues of Theorem B and Theorem A (3) respectively. In [RTZ13, they are proved using Zink's theory of windows and displays of $p$-divisible groups and involve rather delicate linear algebra computation. In contrast, in our method we rarely directly work with $p$-divisible groups and we completely avoid computations with windows or displays. Instead we make use of what are essentially consequences of Kisin's construction of integral models of Hodge type Shimura varieties to abstractly reduce the problem to algebraic geometry over $k$. More specifically, we reduce the intersection length computation to the study of a certain scheme of the form $S_{\Lambda}^{\bar{g}}$ (Proposition 5.1.4), where $S_{\Lambda}$ is a smooth projective $k$-variety closely related to orthogonal Grassmannians, and $\bar{g}$ is a 
certain finite order automorphism of $S$. Thus our method overcomes the difficulty of non-PEL type and also makes the actual computation much more elementary.

It is worth mentioning that our method also applies to the unitary case considered in [RTZ13]. Even in this PEL type case, our method gives a new and arguably simpler proof of the arithmetic fundamental lemma in the minuscule case. This will be pursued in a forthcoming work.

It is also worth mentioning that the very recent work of Bueltel-Pappas [BP17] gives a new moduli interpretation for Rapoport-Zink spaces of Hodge type when restricted to $p$-nilpotent noetherian algebras. Their moduli description is purely group-theoretic (in terms of $(G, \mu)$-displays) and does not involve $p$ divisible groups. Although we do not use $(G, \mu)$-displays in this article, it would be interesting to see if it is possible to extend the results of this article using their group-theoretic description (e.g., to non-minuscule cases).

1.4. Strategy of the proofs. Our key observation is that in order to prove these theorems, we only need to understand $\mathcal{O}$-points of RZ for very special choices of $W$-algebras $\mathcal{O}$.

To prove Theorem B, it turns out that we only need to understand $\mathrm{RZ}\left(W / p^{2}\right)$ and $\mathrm{RZ}\left(k[\epsilon] / \epsilon^{2}\right)$. Note that the $W$-algebras $W / p^{2}$ and $k[\epsilon] / \epsilon^{2}$, when viewed as thickenings of $\operatorname{Spec} k$ (under reduction modulo $p$ or $\epsilon$ respectively), are objects of the crystalline site of Spec $k$. For such an object $\mathcal{O}$, we prove in Theorem 4.1 .7 an explicit description of $\operatorname{RZ}(\mathcal{O})$ and more generally an explicit description of $\mathcal{Z}(\mathcal{O})$, for any special cycle $\mathcal{Z}$ in RZ. Theorem 4.1.7 is the main tool to prove Theorem B, and is also the only place we use $p$-divisible groups. This result is a Rapoport-Zink space analogue of a result of Madapusi Pera [MP16, Proposition 5.16] for GSpin Shimura varieties. Its proof also relies on loc. cit. and is ultimately a consequence of Kisin's construction of the integral canonical models of Hodge type Shimura varieties [Kis10.

To prove the intersection length formula Theorem A (3), let $\Lambda$ be the vertex lattice $L(g)^{\vee}$. Theorem B allows us to reduce Theorem A (3) to the problem of studying the fixed-point subscheme of the smooth $k$-variety $S_{\Lambda} \cong p^{\mathbb{Z}} \backslash \mathrm{RZ}_{\Lambda}^{\text {red }}$, under the induced action $\bar{g} \in \mathrm{SO}\left(\Lambda / \Lambda^{\vee}\right)$ of $g$. Since the fixed point of a smooth $k$-variety under a group of order coprime to $p$ is still smooth ([Ive72, 1.3]), this point of view immediately explains that when $\bar{g}$ is semisimple (in which case $m(Q(T))=1$ ), the intersection multiplicity must be 1 . More generally, we utilize Howard-Pappas's description of $S_{\Lambda}$ in [HP15] and reduce the intersection length computation to elementary algebraic geometry of orthogonal Grassmannians over $k$ (Proposition 5.3 .5 and Theorem 5.4.1).

The remaining parts of Theorem A are relatively easier. From Theorem B it is not difficult to deduce Theorem A (1). The set of $k$-points of RZ is well understood group theoretically in terms of the affine Deligne-Lusztig set. The point counting formula Theorem A (2) essentially only relies on this description, and we follow the strategy in [RTZ13] to give a short streamlined proof (Proposition 3.4.4).

1.5. Organization of the paper. In $\$ 2$, we review the structure of GSpin Rapoport-Zink spaces and special cycles. In \$3, we formulate the arithmetic intersection problem of GGP cycles and prove the pointcounting formula for the $k$-points of the intersection in the minuscule case (Theorem A (2)). In \&4, we prove reducedness of minuscule special cycles (Theorem B). In \$5, we deduce from Theorem B that the arithmetic intersection is concentrated in the special fiber (Theorem A (1)) and finally compute the intersection length when $p$ is sufficiently large (Theorem A (3)).

1.6. Acknowledgments. We are very grateful to B. Howard, M. Kisin, M. Rapoport and W. Zhang for helpful conversations or comments. Our debt to the two papers [RTZ13] and [HP15] should be clear to the readers. 


\section{GSPIN RAPOPORT-ZinK SPACES}

In this section we review the structure of GSpin Rapoport-Zink spaces due to Howard-Pappas [HP15]. We refer to [HP15] for the proofs of these facts.

2.1. Quadratic spaces and GSpin groups. Let $p$ be an odd prime. Let $(V, q)$ be a non-degenerate selfdual quadratic space over $\mathbb{Z}_{p}$ of rank $n \geq 3$. By definition the Clifford algebra $C(V)$ is the quotient of the tensor algebra $V^{\otimes}$ by the two sided ideal generated by elements of the form $v \otimes v-q(v)$. It is free of rank $2^{n}$ over $\mathbb{Z}_{p}$. The linear map $v \mapsto-v$ preserves the quadratic form $q$ on $V$ and induces an involution on $C(V)$. This involution decomposes $C(V)=C^{+}(V) \oplus C^{-}(V)$ into even and odd parts. The image of the injection $V \hookrightarrow C^{-}(V)$ generates $C(V)$ as a $\mathbb{Z}_{p}$-algebra.

We also have a canonical involution $*: C(V) \rightarrow C(V)$, which a $\mathbb{Z}_{p}$-linear endomorphism characterized by $\left(v_{1} v_{2} \cdots v_{k}\right)^{*}=v_{k} \cdots v_{2} v_{1}$ for $v_{i} \in V$. The spinor similitude group $G=\operatorname{GSpin}(V)$ is the reductive group over $\mathbb{Z}_{p}$ such that for a $\mathbb{Z}_{p}$-algebra $R$,

$$
G(R)=\left\{g \in C^{+}(V)^{\times}: g V_{R} g^{-1}=V_{R}, \quad g^{*} g \in R^{\times}\right\} .
$$

The character $\eta_{G}: G \rightarrow \mathbb{G}_{m}$ given by $g \mapsto g^{*} g$ is the called spinor similitude.

The conjugation action $g . v=g v g^{-1}$ of $G$ on $C(V)$ stabilizes $V$ and preserves the quadratic form $q$. This action thus defines a homomorphism

$$
G \rightarrow \mathrm{SO}(V)
$$

The kernel of the above morphism is the central $\mathbb{G}_{m}$ inside $G$ given by the natural inclusion $R^{\times} \subset G(R)$ for any $\mathbb{Z}_{p}$-algebra $R$. The restriction of $\eta_{G}$ on the central $\mathbb{G}_{m}$ is given by $g \mapsto g^{2}$. Note that the central $\mathbb{G}_{m}$ in $G$ is equal to the identity component of the center of $G$, and it is equal to the center of $G$ precisely when $n$ is odd.

2.2. Basic elements in GSpin groups. Let $k=\overline{\mathbb{F}}_{p}, W=W(k)$ and $K=W[1 / p]$. Let $\sigma \in \operatorname{Aut}(W)$ be the lift of the absolute $p$-Frobenius on $k$. Let $D=\operatorname{Hom}_{\mathbb{Z}_{p}}\left(C(V), \mathbb{Z}_{p}\right)$ be the contragredient $G$-representation of $C(V)$.

Any $b \in G(K)$ determines two isocrystals

$$
\left(V_{K}, \Phi=b \circ \sigma\right), \quad\left(D_{K}, F=b \circ \sigma\right) .
$$

Denote by $\mathbb{T}$ the pro-torus over $\mathbb{Q}_{p}$ of character group $\mathbb{Q}$. Recall that $b \in G(K)$ is basic if its slope morphism $\nu_{b}: \mathbb{T}_{K} \rightarrow G_{K}$ factors through (the identity component) of $Z\left(G_{K}\right)$, i.e., factors through the central $\mathbb{G}_{m}$. By [HP15, 4.2.4], $b$ is basic if and only if $\left(V_{K}, \Phi\right)$ is isoclinic of slope 0 , if and only if $\left(D_{K}, F\right)$ is isoclinic of slope $-\nu_{b} \in \operatorname{Hom}\left(\mathbb{T}_{K}, \mathbb{G}_{m}\right) \cong \mathbb{Q}$. The map $b \mapsto \nu_{b}$ gives a bijection between the set of basic $\sigma$-conjugacy classes and the set $\frac{1}{2} \mathbb{Z}$. Moreover, the $\mathbb{Q}_{p}$-quadratic space

$$
V_{K}^{\Phi}=\left\{x \in V_{K}: \Phi x=x\right\}
$$

has the same dimension and determinant as $V_{\mathbb{Q}_{p}}$, and has Hasse invariant $(-1)^{2 \nu_{b}}([$ HP15, 4.2.5])).

2.3. Local unramified Shimura-Hodge data. Since $V$ is self-dual, we know that $V_{\mathbb{Q}_{p}}$ has Hasse invariant +1 . In particular $V$ contains at least one hyperbolic plane and we can pick a $\mathbb{Z}_{p}$-basis $x_{1}, \ldots, x_{n}$ of $V$ such 
that the Gram matrix of the quadratic form $q$ is of the form

$$
\left(\begin{array}{cccccc}
0 & 1 & & & & \\
1 & 0 & & & & \\
& & * & & & \\
& & & * & & \\
& & & & \ddots & \\
& & & & & *
\end{array}\right)
$$

We will fix $x_{1}, \ldots, x_{n}$ once and for all. Define a cocharacter

$$
\mu: \mathbb{G}_{m} \rightarrow G, \quad t \mapsto t^{-1} x_{1} x_{2}+x_{2} x_{1} .
$$

Pick an explicit element $b=x_{3}\left(p^{-1} x_{1}+x_{2}\right) \in G\left(\mathbb{Q}_{p}\right)$, then one can show that $b$ is basic with $\nu_{b}=\frac{1}{2}$. Thus $V_{K}^{\Phi}$ has the opposite Hasse invariant -1 (cf. 2.2 ).

Fix any $\delta \in C(V)^{\times}$such that $\delta^{*}=-\delta$. Then $\psi_{\delta}\left(c_{1}, c_{2}\right)=\operatorname{Trd}\left(c_{1} \delta c_{2}^{*}\right)$ defines a non-degenerate symplectic form on $C(V)$, where Trd : $C(V) \rightarrow \mathbb{Z}_{p}$ is the reduced trace. We have a closed immersion into the symplectic similitude group

$$
G \hookrightarrow \operatorname{GSp}\left(C(V), \psi_{\delta}\right)
$$

By [HP15, 4.2.6], the tuple $(G, b, \mu, C(V))$ defines a local unramified Shimura-Hodge datum (in the sense of [HP15, 2.2.4]). In fact, for the fixed $G$ and $\mu$, the $\sigma$-conjugacy class of $b$ is the unique basic $\sigma$-conjugacy class for which $(G, b, \mu)$ is a local unramified Shimura-Hodge datum (cf. [HP15, 4.2.7]).

Remark 2.3.1. The tuple $(G, b, \mu, C(V))$ is chosen in such a way that the associated Rapoport-Zink space (see below) provides a $p$-adic uniformization for the supersingular locus of a related GSpin Shimura variety. For more details on the relation with Shimura varieties see [HP15, §7].

2.4. GSpin Rapoport-Zink spaces. There is a unique (up to isomorphism) $p$-divisible group $\mathbb{X}_{0} / k$ such that its (contravariant) Dieudonné module $\mathbb{D}\left(\mathbb{X}_{0}\right)$ is given by the $W$-lattice $D_{W}$ in the isocrystal $D_{K}$. The non-degenerate symplectic form $\psi_{\delta}$ induces a principal polarization $\lambda_{0}$ of $\mathbb{X}_{0}$. Fix a collection of tensors $\left(s_{\alpha}\right)$ on $C(V)$ cutting out $G$ from $\mathrm{GL}(C(V))$ (including the symplectic form $\psi_{\delta}$ ). By [HP15, 4.2.7], we have a GSpin Rapoport-Zink space

$$
\mathrm{RZ}:=\mathrm{RZ}\left(G, b, \mu, C(V),\left(s_{\alpha}\right)\right) .
$$

It is a formal scheme over $W$, together with a closed immersion into the symplectic Rapoport-Zink space $\mathrm{RZ}\left(\mathbb{X}_{0}, \lambda_{0}\right)$. Moreover, the formal scheme $\mathrm{RZ}$ itself depends only on the local unramified Shimura-Hodge datum $(G, b, \mu, C(V))$, and not on the choices of the tensors $\left(s_{\alpha}\right)$.

Denote by $(X, \rho, \lambda)$ the universal triple over $\mathrm{RZ}\left(\mathbb{X}_{0}, \lambda_{0}\right)$, where $X$ is the universal $p$-divisible group, $\rho$ is the universal quasi-isogeny, and $\lambda$ is the universal polarization. Consider the restriction of this triple to the closed formal subscheme $\mathrm{RZ}$ of $\mathrm{RZ}\left(\mathbb{X}_{0}, \lambda_{0}\right)$. We denote this last triple also by $(X, \rho, \lambda)$ and call it the universal triple over RZ.

Remark 2.4.1. Let $\mathrm{Nilp}_{W}$ be the category of $W$-algebras in which $p$ is nilpotent. As a set-valued functor on the category Nilp $_{W}$, the symplectic Rapoport-Zink space $\mathrm{RZ}\left(\mathbb{X}_{0}, \lambda_{0}\right)$ has an explicit moduli interpretation in terms of triples $(X, \rho, \lambda)$. In contrast, the subfunctor defined by RZ does not have an explicit description. In fact, in [HP15] Howard-Pappas only give a moduli interpretation of RZ when it is viewed as a set-valued functor on a more restricted category $\mathrm{ANilp}_{\mathrm{W}}^{\mathrm{fsm}}$. In this article we do not make use of this last moduli interpretation. All we will need is the global construction of RZ as a formal subscheme of $\mathrm{RZ}\left(\mathbb{X}_{0}, \lambda_{0}\right)$ due to Howard-Pappas. 
Over RZ, the universal quasi-isogeny $\rho$ respects the polarizations $\lambda$ and $\lambda_{0}$ up to a scalar $c(\rho) \in \mathbb{Q}_{p}^{\times}$, i.e., $\rho^{\vee} \circ \lambda \circ \rho=c^{-1}(\rho) \cdot \lambda_{0}$ (Zariski locally on $\mathrm{RZ}_{k}$ ). Let $\mathrm{RZ}{ }^{(\ell)} \subseteq \mathrm{RZ}$ be the closed and open formal subscheme where $\operatorname{ord}_{p}(c(\rho))=\ell$. We have the decomposition into a disjoint union

$$
\mathrm{RZ}=\coprod_{\ell \in \mathbb{Z}} \mathrm{RZ}^{(\ell)}
$$

In fact each $\mathrm{RZ}^{(\ell)}$ is connected and they are mutually (non-canonically) isomorphic. cf. [HP15, 4.3.3, 4.3.4].

2.5. The group $J_{b}$. The algebraic group $J_{b}=\operatorname{GSpin}\left(V_{K}^{\Phi}\right)$ has $\mathbb{Q}_{p}$-points

$$
J_{b}\left(\mathbb{Q}_{p}\right)=\{g \in G(K): g b=b \sigma(g)\},
$$

and $J_{b}\left(\mathbb{Q}_{p}\right)$ acts on $\mathrm{RZ}$ via its action on $\mathbb{X}_{0}$ as quasi-endomorphisms. The action of $g \in J_{b}\left(\mathbb{Q}_{p}\right)$ on RZ restricts to isomorphisms

$$
\mathrm{RZ}^{(\ell)} \stackrel{\sim}{\longrightarrow} \mathrm{RZ}^{\left(\ell+\operatorname{ord}_{p}\left(\eta_{b}(g)\right)\right)}, \ell \in \mathbb{Z}
$$

where $\eta_{b}: J_{b}\left(\mathbb{Q}_{p}\right) \rightarrow \mathbb{Q}_{p}^{\times}$is the spinor similitude. In particular, $p^{\mathbb{Z}} \subseteq J_{b}\left(\mathbb{Q}_{p}\right)$ acts on RZ and since $\eta_{b}(p)=p^{2}$, we have an isomorphism

$$
p^{\mathbb{Z}} \backslash \mathrm{RZ} \cong \mathrm{RZ} \mathrm{Z}^{(0)} \coprod \mathrm{RZ} \mathrm{(1)}^{(1)} .
$$

Remark 2.5.1. In this article we are interested in studying the fixed locus $\mathrm{RZ}^{g}$ of RZ under $g \in J_{b}\left(\mathbb{Q}_{p}\right)$. By (2.5.0.1) this is non-empty only when $\operatorname{ord}_{p}\left(\eta_{b}(g)\right)=0$. Since $p^{\mathbb{Z}}$ is central in $J_{b}\left(\mathbb{Q}_{p}\right)$, one could also study $\left(p^{\mathbb{Z}} \backslash \mathrm{RZ}\right)^{g}$ for $g \in J_{b}\left(\mathbb{Q}_{p}\right)$. However by (2.5.0.1), we know that $\left(p^{\mathbb{Z}} \backslash \mathrm{RZ}\right)^{g} \neq \varnothing$ only if $\operatorname{ord}_{p}\left(\eta_{b}(g)\right)$ is even, and in this case

$$
\left(p^{\mathbb{Z}} \backslash \mathrm{RZ}\right)^{g} \cong p^{\mathbb{Z}} \backslash \mathrm{RZ}{ }^{g_{0}},
$$

where $g_{0}=p^{-\operatorname{ord}_{p}\left(\eta_{b}(g)\right) / 2} g$. Hence the study of $\left(p^{\mathbb{Z}} \backslash \mathrm{RZ}\right)^{g}$ for general $g$ reduces to the study of RZ ${ }^{g}$ for $g$ satisfying $\operatorname{ord}_{p}\left(\eta_{b}(g)\right)=0$.

2.6. Special endomorphisms. Using the injection $V \hookrightarrow C(V)^{\mathrm{op}}$, we can view

$$
V \subseteq \operatorname{End}_{\mathbb{Z}_{p}}(D)
$$

as special endomorphisms of $D$ : the action of $v \in V$ on $D$ is explicitly given by

$$
(v d)(c)=d(v c), \quad d \in D, c \in C(V) .
$$

Base changing to $K$ gives $V_{K} \subseteq \operatorname{End}_{K}\left(D_{K}\right)$. Since the $F$-equivariant endomorphisms $\operatorname{End}_{K, F}\left(D_{K}\right)$ can be identified with the space of quasi-endomorphisms $\operatorname{End}^{0}\left(\mathbb{X}_{0}\right)$ of $\mathbb{X}_{0}$, we obtain an embedding of $\mathbb{Q}_{p}$-vector spaces

$$
V_{K}^{\Phi} \hookrightarrow \operatorname{End}^{0}\left(\mathbb{X}_{0}\right)
$$

Elements of $V_{K}^{\Phi}$ are thus viewed as quasi-endomorphisms of $\mathbb{X}_{0}$, and we call them special quasi-endomorphisms.

\subsection{Vertex lattices.}

Definition 2.7.1. A vertex lattice is a $\mathbb{Z}_{p}$-lattice $\Lambda \subseteq V_{K}^{\Phi}$ such that

$$
p \Lambda \subseteq \Lambda^{\vee} \subseteq \Lambda .
$$

We define

$$
\Omega_{0}=\Lambda / \Lambda^{\vee} .
$$

Then the quadratic form $v \mapsto p \cdot q(v)$ makes $\Omega_{0}$ a non-degenerate quadratic space over $\mathbb{F}_{p}$. The type of $\Lambda$ is defined to be $t_{\Lambda}:=\operatorname{dim}_{\mathbb{F}_{p}} \Omega_{0}$. 
By [HP15, 5.1.2], the type of a vertex lattice is always an even integer such that $2 \leq t_{\Lambda} \leq t_{\max }$, where

$$
t_{\max }= \begin{cases}n-2, & \text { if } n \text { is even and } \operatorname{det}\left(V_{\mathbb{Q}_{p}}\right)=(-1)^{n / 2} \in \mathbb{Q}_{p}^{\times} /\left(\mathbb{Q}_{p}^{\times}\right)^{2}, \\ n-1, & \text { if } n \text { is odd, } \\ n, & \text { if } n \text { is even and } \operatorname{det}\left(V_{\mathbb{Q}_{p}}\right) \neq(-1)^{n / 2} \in \mathbb{Q}_{p}^{\times} /\left(\mathbb{Q}_{p}^{\times}\right)^{2} .\end{cases}
$$

It follows that the quadratic space $\Omega_{0}$ is always non-split, because otherwise a Lagrangian subspace $\mathcal{L} \subseteq \Omega_{0}$ would provide a vertex lattice $\Lambda^{\vee}+\mathcal{L} \subseteq V_{K}^{\Phi}$ of type 0 (cf. [HP15, 5.3.1])

\subsection{The variety $S_{\Lambda}$.}

Definition 2.8.1. Define

$$
\Omega=\Omega_{0} \otimes_{\mathbb{F}_{p}} k \cong \Lambda_{W} / \Lambda_{W}^{\vee}
$$

Let $d=t_{\Lambda} / 2$. Let $\operatorname{OGr}(\Omega)$ be the moduli space of Lagrangian subspaces $\mathcal{L} \subseteq \Omega$. We define $S_{\Lambda} \subseteq \operatorname{OGr}(\Omega)$ to be the reduced closed subscheme of $\operatorname{OGr}(\Omega)$ with $k$-points given as follows:

$$
\begin{aligned}
S_{\Lambda}(k) & =\{\text { Lagrangian subspaces } \mathcal{L} \subseteq \Omega: \operatorname{dim}(\mathcal{L}+\Phi(\mathcal{L}))=d+1\} \\
& \cong\left\{\left(\mathcal{L}_{d-1}, \mathcal{L}_{d}\right): \mathcal{L}_{d} \subseteq \Omega \text { Lagrangian, } \mathcal{L}_{d-1} \subseteq \mathcal{L}_{d} \cap \Phi \mathcal{L}_{d}, \operatorname{dim} \mathcal{L}_{d-1}=d-1\right\},
\end{aligned}
$$

where the last bijection is given by $\mathcal{L} \mapsto(\mathcal{L} \cap \Phi \mathcal{L}, \mathcal{L})$.

More precisely, for any $k$-algebra $R$, the $R$-points $S_{\Lambda}(R)$ is the set of pairs $\left(\mathcal{L}_{d-1}, \mathcal{L}_{d}\right)$ such that:

- $\mathcal{L}_{d}$ is a totally isotropic $R$-submodule of $\Omega \otimes_{k} R$ that is an $R$-module local direct summand of $\Omega \otimes_{k} R$ and of local rank $d$,

- $\mathcal{L}_{d-1}$ is an $R$-module local direct summand of $\Omega \otimes_{k} R$ and of local rank $d-1$,

- $\mathcal{L}_{d-1} \subset \mathcal{L}_{d} \cap \Phi \mathcal{L}_{d}$, where $\Phi$ acts on $\Omega \otimes_{k} R$ via the $p$-Frobenius on $R$. In particular, $\mathcal{L}_{d-1}$ is totally isotropic, and is a local direct summand of $\mathcal{L}_{d}$ and of $\Phi \mathcal{L}_{d}$. (For the last statement see Remark 2.8.2 below.)

By [HP15, 5.3.2], $S_{\Lambda}$ is a $k$-variety with two isomorphic connected components $S_{\Lambda}^{ \pm}$, each being projective and smooth of dimension $t_{\Lambda} / 2-1$. For more details, see [HP15, §5.3] and [HP14, §3.2].

Remark 2.8.2. In the sequel we will frequently use the following simple fact without explicitly mentioning it. Let $R$ be a commutative ring and $M$ a free $R$-module of finite rank. Suppose $M_{1}, M_{2}$ are submodules of $M$ that are local direct summands. Suppose $M_{1} \subset M_{2}$. Then $M_{1}$ is a local direct summand of $M_{2}$, and both $M_{1}$ and $M_{2}$ are locally free.

\subsection{Structure of the reduced scheme $\mathrm{RZ}^{\mathrm{red}}$.}

Definition 2.9.1. For a vertex lattice $\Lambda$, we define $\mathrm{RZ} \Lambda \subseteq \mathrm{RZ}$ to be locus where $\rho \circ \Lambda^{\vee} \circ \rho^{-1} \subseteq \operatorname{End}(X)$, i.e. the quasi-endomorphisms $\rho \circ v \circ \rho^{-1}$ lift to actual endomorphisms for any $v \in \Lambda^{\vee}$. In other words, if we define a locus $\mathrm{RZ}\left(\mathbb{X}_{0}, \lambda_{0}\right)_{\Lambda}$ using the same condition inside $\mathrm{RZ}\left(\mathbb{X}_{0}, \lambda_{0}\right)$ (a closed formal subscheme by [RZ96, Proposition 2.9]), then $\mathrm{RZ}_{\Lambda}$ is the intersection of $\mathrm{RZ}$ with $\mathrm{RZ}\left(\mathbb{X}_{0}, \lambda_{0}\right)_{\Lambda}$ inside $\mathrm{RZ}\left(\mathbb{X}_{0}, \lambda_{0}\right)$. In particular, $\mathrm{RZ}_{\Lambda}$ is a closed formal subscheme of RZ.

Consider the reduced subscheme $\mathrm{RZ}^{(\ell), \text { red }}$ of $\mathrm{RZ}^{(\ell)}$. By the result [HP15, 6.4.1], the irreducible components of $\mathrm{RZ}^{(\ell) \text {,red }}$ are precisely $\mathrm{RZ}_{\Lambda}^{(\ell) \text {,red }}$, where $\Lambda$ runs through the vertex lattices of the maximal type $t_{\Lambda}=t_{\max }$. Moreover, there is an isomorphism of $k$-schemes ([HP15, 6.3.1])

$$
p^{\mathbb{Z}} \backslash \mathrm{RZ} Z_{\Lambda}^{\text {red }} \stackrel{\sim}{\longrightarrow} S_{\Lambda},
$$

which also induces an isomorphism between $\mathrm{RZ}_{\Lambda}^{(\ell) \text {,red }}$ and $S_{\Lambda}^{ \pm}$, for each $\ell \in \mathbb{Z}$. In particular, $\mathrm{RZ}^{\text {red }}$ is equidimensional of dimension $t_{\max } / 2-1$. 
2.10. The Bruhat-Tits stratification. For any vertex lattices $\Lambda_{1}$ and $\Lambda_{2}$, the intersection $R Z_{\Lambda_{1}}^{\text {red }} \cap R Z_{\Lambda_{2}}^{\text {red }}$ is nonempty if and only if $\Lambda_{1} \cap \Lambda_{2}$ is also a vertex lattice, in which case it is equal to $R Z_{\Lambda_{1} \cap \Lambda_{2}}^{\text {red }}$ ([HP15, 6.2.4]). In this way we obtain a Bruhat-Tits stratification on $\mathrm{RZ}^{\mathrm{red}}$. Associated to a vertex lattice $\Lambda$, we define an open subscheme of $\mathrm{RZ}_{\Lambda}^{\mathrm{red}}$ given by

$$
\mathrm{BT}_{\Lambda}=\mathrm{RZ}_{\Lambda}^{\mathrm{red}}-\bigcup_{\Lambda^{\prime} \subsetneq \Lambda} \mathrm{RZ}_{\Lambda^{\prime}}^{\mathrm{red}}
$$

Then

$$
\mathrm{RZ}^{\mathrm{red}}=\coprod_{\Lambda} \mathrm{BT}_{\Lambda}
$$

is a disjoint union of locally closed subschemes, indexed by all vertex lattices.

2.11. Special lattices. One can further parametrize the $k$-points in each $\mathrm{RZ}_{\Lambda}$ using special lattices.

Definition 2.11.1. We say a $W$-lattice $L \subseteq V_{K}$ is a special lattice if $L$ is self-dual and $(L+\Phi(L)) / L \cong W / p W$.

We have a bijection ([HP15, 6.2.2])

$$
p^{\mathbb{Z}} \backslash \mathrm{RZ}(k) \stackrel{\sim}{\longrightarrow}\left\{\text { special lattices } L \subseteq V_{K}\right\} .
$$

To construct this bijection, one uses the fact ([ Deligne-Lusztig set

$$
X_{G, b, \mu^{\sigma}}(k)=\left\{g \in G(K): g^{-1} b \sigma(g) \in G(W) \mu^{\sigma}(p) G(W)\right\} / G(W) .
$$

The special lattice associated to $g \in G(K)$ is then given by $g \mu\left(p^{-1}\right) \cdot V_{W} \subseteq V_{K}$. Conversely, given a special lattice $L \subseteq V_{K}$, then there exists some $g \in G(K)$ such that $g \mu\left(p^{-1}\right) \cdot V_{W}=L$ and $g \cdot V_{W}=\Phi(L)$. The point in $\mathrm{RZ}(k)$ then corresponds to the image of $g$ in $X_{G, b, \mu^{\sigma}}(k)$. The Dieudonné module of the $p$-divisible group at this point is given by $M=g D_{W} \subseteq D_{K}$ and the image of Verschiebung is $\left(F^{-1} p\right) M=g \cdot p \mu\left(p^{-1}\right) D_{W}$.

Remark 2.11.2. Suppose $x_{0} \in \mathrm{RZ}(k)$ corresponds to the special lattice $L$ under (2.11.1.1). Let $M=\mathbb{D}\left(X_{0}\right) \subset$ $D_{K}$ be the Dieudonné module of the $p$-divisible group $X_{0}$ corresponding to $x_{0}$. Then we have (cf. HP15, $\S 6.2])$

$$
L=\left\{v \in V_{K} \mid v\left(F^{-1} p\right) M \subset\left(F^{-1} p\right) M\right\}, \quad \Phi L=\left\{v \in V_{K} \mid v M \subset M\right\} .
$$

Here we view $V_{K} \subset \operatorname{End}_{K}\left(D_{K}\right)$ as in $\$ 2.6$.

2.12. Special lattices and vertex lattices. For any vertex lattice $\Lambda$, the bijection (2.11.1.1) induces a bijection

$$
p^{\mathbb{Z}} \backslash \mathrm{RZ}_{\Lambda}(k) \stackrel{\sim}{\longrightarrow}\left\{\text { special lattices } L \subseteq V_{K}: \Lambda_{W}^{\vee} \subseteq L \subseteq \Lambda_{W}\right\}=\left\{\text { special lattices } L \subseteq V_{K}: \Lambda_{W}^{\vee} \subseteq L\right\}
$$

Sending a special lattice $L$ to $\mathcal{L}:=L / \Lambda_{W}^{\vee} \subseteq \Omega$ gives a bijection between the right hand side of (2.12.0.1) and $S_{\Lambda}(k)$, which is the effect of the isomorphism (2.9.1.1) on $k$-points.

Definition 2.12.1. For each special lattice $L \subseteq V_{K}$, there is a unique minimal vertex lattice $\Lambda(L) \subseteq V_{K}^{\Phi}$ such that

$$
\Lambda(L)_{W}^{\vee} \subseteq L \subseteq \Lambda(L)_{W}
$$

In fact, let $L^{(r)}=L+\Phi(L)+\cdots+\Phi^{r}(L)$. Then there exists a unique integer $1 \leq d \leq t_{\max } / 2$ such that $L^{(i)} \subsetneq L^{(i+1)}$ for $i<d$, and $L^{(d)}=L^{(d+1)}$. Then $L^{(i+1)} / L^{(i)}$ all have $W$-length 1 for $i<d$, and

$$
\Lambda(L):=\left(L^{(d)}\right)^{\Phi} \subseteq V_{K}^{\Phi}
$$

is a vertex lattice of type $2 d$ and $\Lambda(L)^{\vee}=L^{\Phi}$. 
Notice that $\Lambda(L)_{W}$ is the smallest $\Phi$-invariant lattice containing $L$ and $\Lambda(L)_{W}^{\vee}$ is the largest $\Phi$-invariant lattice contained in $L$. It follows that the element of $\mathrm{RZ}(k)$ corresponding to a special lattice $L$ lies in $\mathrm{RZ}_{\Lambda}$ if and only if $\Lambda(L) \subseteq \Lambda$, and it lies in $\mathrm{BT}_{\Lambda}$ if and only if $\Lambda(L)=\Lambda$. Thus we have the bijection

$$
p^{\mathbb{Z}} \backslash \mathrm{BT}_{\Lambda}(k) \stackrel{\sim}{\longrightarrow}\{L \text { special lattices }: \Lambda(L)=\Lambda\} .
$$

2.13. Deligne-Lusztig varieties. For any vertex lattice $\Lambda$, by [HP15, 6.5.6], $p^{\mathbb{Z}} \backslash \mathrm{BT}_{\Lambda}$ is a smooth quasiprojective variety of dimension $t_{\Lambda} / 2-1$, isomorphic to a disjoint union of two Deligne-Lusztig varieties $X_{B}\left(w^{ \pm}\right)$associated to two Coxeter elements $w^{ \pm}$in the Weyl group of $\operatorname{SO}\left(\Omega_{0}\right)$. Here $\Omega_{0}:=\Lambda / \Lambda^{\vee}$ is the quadratic space over $\mathbb{F}_{p}$ defined in Definition 2.7.1. In particular, the $k$-variety $p^{\mathbb{Z}} \backslash \mathrm{BT}_{\Lambda}$ only depends on the quadratic space $\Omega_{0}$.

Let us recall the definition of $X_{B}\left(w^{ \pm}\right)$. Let $d=t_{\Lambda} / 2$. Let $\langle\cdot, \cdot\rangle$ be the bilinear pairing on $\Omega_{0}$. Since $\Omega_{0}$ is a non-degenerate non-split quadratic space over $\mathbb{F}_{p}$ (\$2.7), one can choose a basis $e_{1}, \ldots, e_{d}, f_{d}, \ldots, f_{1}$ of $\Omega$ such that $\left\langle e_{i}, f_{i}\right\rangle=1$ and all other pairings between the basis vectors are 0 , and $\Phi$ fixes $e_{i}, f_{i}$ for $i=1, \ldots, d-1$ and interchanges $e_{d}$ with $f_{d}$. This choice of basis gives a maximal $\Phi$-stable torus $T \subseteq \mathrm{SO}(\Omega)$ (diagonal under this basis), and a $\Phi$-stable Borel subgroup $B \supseteq T$ as the common stabilizer of the two complete isotropic flags

$$
\mathcal{F}^{ \pm}:\left\langle e_{1}\right\rangle \subseteq\left\langle e_{1}, e_{2}\right\rangle \subseteq \cdots \subseteq\left\langle e_{1}, \ldots, e_{d-1}, e_{d}^{ \pm}\right\rangle,
$$

where $e_{d}^{+}:=e_{d}$ and $e_{d}^{-}:=f_{d}$. Let $s_{i}(i=1, \ldots, d-2)$ be the reflection $e_{i} \leftrightarrow e_{i+1}, f_{i} \leftrightarrow f_{i+1}$ and let $t^{ \pm}$be the reflection $e_{d-1} \leftrightarrow e_{d}^{ \pm}, f_{d-1} \leftrightarrow e_{d}^{\mp}$. Then the Weyl group $W(T)=N(T) / T$ is generated by $s_{1}, \cdots, s_{d-2}, t^{+}, t^{-}$. We also know that $W(T)$ sits in a split exact sequence

$$
0 \rightarrow(\mathbb{Z} / 2 \mathbb{Z})^{d-1} \rightarrow W(T) \rightarrow S_{d} \rightarrow 0 .
$$

Since $\Phi$ fixes $s_{i}$ and swaps $t^{+}$and $t^{-}$, we know the $d-1$ elements $s_{1}, \ldots, s_{d-2}, t^{+}\left(\right.$resp. $\left.s_{1}, \ldots, s_{d-2}, t^{-}\right)$ form a set of representatives of $\Phi$-orbits of the simple reflections. Therefore

$$
w^{ \pm}:=t^{\mp} s_{d-2} \cdots s_{2} s_{1} \in W(T)
$$

are Coxeter elements of minimal length. The Deligne-Lusztig variety associated to $B$ and the Coxeter element $w^{ \pm}$is defined to be

$$
X_{B}\left(w^{ \pm}\right):=\left\{g \in \mathrm{SO}(\Omega) / B: \operatorname{inv}(g, \Phi(g))=w^{ \pm}\right\},
$$

where $\operatorname{inv}(g, h) \in B \backslash \mathrm{SO}(\Omega) / B \cong W(T)$ is the relative position between the two Borels $g B g^{-1}$ and $h B h^{-1}$. The variety $X_{B}\left(w^{ \pm}\right)$has dimension $d-1$. Under the map $g \mapsto g \mathcal{F}^{ \pm}$, the disjoint union $X_{B}\left(w^{+}\right) \amalg X_{B}\left(w^{-}\right)$ can be identified with the variety of complete isotropic flags

$$
\mathcal{F}: \mathcal{F}_{1} \subseteq \mathcal{F}_{2} \subseteq \cdots \subseteq \mathcal{F}_{d}
$$

such that $\mathcal{F}_{i}=\mathcal{F}_{i-1}+\Phi\left(\mathcal{F}_{i-1}\right)$ and $\operatorname{dim}_{k}\left(\mathcal{F}_{d}+\Phi\left(\mathcal{F}_{d}\right)\right)=d+1$. The two components are interchanged by an orthogonal transformation of determinant -1 . Notice that such $\mathcal{F}$ is determined by the isotropic line $\mathcal{F}_{1}$ by

$$
\mathcal{F}_{i}=\mathcal{F}_{1}+\Phi\left(F_{1}\right) \cdots+\Phi^{i-1}\left(\mathcal{F}_{1}\right)
$$

and is also determined by the Lagrangian $\mathcal{F}_{d}$ by

$$
\mathcal{F}_{i}=\mathcal{F}_{d} \cap \Phi\left(\mathcal{F}_{d}\right) \cap \cdots \cap \Phi^{d-i}\left(\mathcal{F}_{d}\right) .
$$

The bijection (2.12.1.1) induces a bijection

$$
p^{\mathbb{Z}} \backslash \mathrm{BT}_{\Lambda}(k) \stackrel{\sim}{\longrightarrow} X_{B}\left(w^{+}\right)(k) \coprod X_{B}\left(w^{-}\right)(k)
$$


by sending a special lattice $L$ with $\Lambda(L)=\Lambda$ to the flag determined by the Lagrangian $\mathcal{F}_{d}=L / \Lambda_{W}^{\vee}$. This bijection is the restriction of the isomorphism (2.9.1.1) on $k$-points and we obtain the desired isomorphism

$$
p^{\mathbb{Z}} \backslash \mathrm{BT}_{\Lambda} \cong X_{B}\left(w^{+}\right) \coprod X_{B}\left(w^{-}\right) .
$$

\subsection{Special cycles.}

Definition 2.14.1. For an $m$-tuple $\mathbf{v}=\left(v_{1}, \ldots, v_{m}\right)$ of vectors in $V_{K}^{\Phi}$, define its fundamental matrix $T(\mathbf{v})=$ $\left(\left\langle v_{i}, v_{j}\right\rangle\right)_{i, j=1, \ldots, m}$. We define the special cycle $\mathcal{Z}(\mathbf{v}) \subseteq \mathrm{RZ}$ to be the locus where $\rho \circ v_{i} \circ \rho^{-1} \in \operatorname{End}(X)$, i.e., all the quasi-endomorphisms $\rho \circ v_{i} \circ \rho^{-1}$ lift to actual endomorphisms on $X(i=1, \ldots, m)$. Similar to Definition 2.9.1, $\mathcal{Z}(\mathbf{v})$ is a closed formal subscheme of RZ, which is the intersection RZ with the analogously defined cycle inside $\mathrm{RZ}\left(\mathbb{X}_{0}, \lambda_{0}\right)$. Since $\mathcal{Z}(\mathbf{v})$ only depends on the $\mathbb{Z}_{p}$-submodule $\operatorname{span}_{\mathbb{Z}_{p}}(\mathbf{v})$ of $V_{K}^{\Phi}$, we also write $\mathcal{Z}\left(\operatorname{span}_{\mathbb{Z}_{p}}(\mathbf{v})\right)$.

Remark 2.14.2. Let $x_{0} \in \mathrm{RZ}(k)$ correspond to $L$ under (2.11.1.1). Let $\mathbf{v}$ be an arbitrary $\mathbb{Z}_{p}$-submodule of $V_{K}^{\Phi}$. By Remark 2.11.2 we know that $x_{0} \in \mathcal{Z}(\mathbf{v})$ if and only if $\mathbf{v} \subset \Phi L$, if and only if $\mathbf{v} \subset \Phi L \cap L$ (as $\mathbf{v}$ is $\Phi$-invariant).

Definition 2.14.3. When $m=n$ and $T(\mathbf{v})$ is non-singular, we obtain a lattice

$$
L(\mathbf{v})=\mathbb{Z}_{p} v_{1}+\cdots \mathbb{Z}_{p} v_{n} \subseteq V_{K}^{\Phi} .
$$

By the Cartan decomposition, $T(\mathbf{v}) \in \mathrm{GL}_{n}\left(\mathbb{Z}_{p}\right) \operatorname{diag}\left(p^{r_{1}}, p^{r_{2}}, \cdots, p^{r_{n}}\right) \mathrm{GL}_{n}\left(\mathbb{Z}_{p}\right)$ for a unique non-increasing sequence of integers $r_{1} \geq \cdots \geq r_{n}$. Note that if we view the matrix $T(\mathbf{v})^{-1}$ as a linear operator $V_{K}^{\Phi} \rightarrow V_{K}^{\Phi}$ using the basis $\mathbf{v}$, it sends $\mathbf{v}$ to the dual basis of $\mathbf{v}$, and in particular it sends any $\mathbb{Z}_{p}$-basis of $L(\mathbf{v})$ to a $\mathbb{Z}_{p}$-basis of $L(\mathbf{v})^{\vee}$. Therefore the tuple $\left(r_{1}, \cdots, r_{n}\right)$ is characterized by the condition that there is a basis

$e_{1}, \ldots, e_{n}$ of $L(\mathbf{v})$ such that $p^{-r_{1}} e_{1}, \ldots, p^{-r_{n}} e_{n}$ form a basis of $L(\mathbf{v})^{\vee}$. From this characterization we also see that the tuple $\left(r_{1}, \cdots, r_{n}\right)$ is an invariant only depending on the lattice $L(\mathbf{v})$. We say $\mathbf{v}$ is minuscule if $T(\mathbf{v})$ is non-singular and $r_{1}=1, r_{n} \geq 0$.

Remark 2.14.4. Suppose $m=n$ and $T(\mathbf{v})$ is non-singular. Then $\mathbf{v}$ is minuscule if and only if $L(\mathbf{v})^{\vee}$ is a vertex lattice. In this case by definition $\mathcal{Z}(\mathbf{v})=\mathrm{RZ}_{L(\mathbf{v})^{v}}$.

\section{The InTERSECTION PROBLEM AND The POINT-COUNTING FORMUlA}

3.1. The GSpin Rapoport-Zink subspace. From now on we assume $n \geq 4$. Suppose the last basis vector $x_{n} \in V$ has norm 1 . Then the quadratic subspace of dimension $n-1$

$$
V^{\mathrm{b}}=\mathbb{Z}_{p} x_{1}+\cdots \mathbb{Z}_{p} x_{n-1}
$$

is also self-dual. Let $G^{b}=\operatorname{GSpin}\left(V^{b}\right)$. Analogously we define the element

$$
b^{b}=x_{3}\left(p^{-1} x_{1}+x_{2}\right) \in G^{b}\left(\mathbb{Q}_{p}\right)
$$

and the cocharacter

$$
\mu^{b}: \mathbb{G}_{m} \rightarrow G^{b}, \quad t \mapsto t^{-1} x_{1} x_{2}+x_{2} x_{1} .
$$

As in $\$ 2.4$, we have an associated GSpin Rapoport-Zink space

$$
\mathrm{RZ}^{b}=\operatorname{RZ}\left(G^{b}, b^{b}, \mu^{b}, C\left(V^{b}\right)\right) .
$$

The embedding $V^{b} \hookrightarrow V$ induces an embedding of Clifford algebras $C\left(V^{b}\right) \hookrightarrow C(V)$ and a closed embedding of group schemes $G^{b} \hookrightarrow G$ over $\mathbb{Z}_{p}$, which maps $b^{b}$ to $b$ and $\mu^{b}$ to $\mu$. Thus by the functoriality of RapoportZink spaces ([Kim13, 4.9.6]), we have a closed immersion

$$
\delta: \mathrm{RZ}^{b} \hookrightarrow \mathrm{RZ}
$$


of formal schemes over $W$.

3.2. Relation with the special divisor $\mathcal{Z}\left(x_{n}\right)$. For compatible choices of symplectic forms $\psi^{b}$ on $C\left(V^{b}\right)$ and $\psi$ on $C(V)$, the closed embedding of group schemes $\operatorname{GSp}\left(C\left(V^{b}\right), \psi^{b}\right) \hookrightarrow \operatorname{GSp}(C(V), \psi)$ induces a closed immersion of symplectic Rapoport-Zink spaces (\$2.4)

$$
\phi: \mathrm{RZ}\left(\mathbb{X}_{0}^{b}, \lambda_{0}^{b}\right) \hookrightarrow \mathrm{RZ}\left(\mathbb{X}_{0}, \lambda_{0}\right)
$$

Since we have a decomposition of $\operatorname{GSp}\left(C\left(V^{b}\right), \psi^{b}\right)$-representations

$$
C(V) \cong C\left(V^{b}\right) \oplus C\left(V^{b}\right) x_{n}
$$

we know the moduli interpretation of $\phi$ is given by sending a triple $\left(X^{b}, \rho^{b}, \lambda^{b}\right)$ to the $p$-divisible group $X=X^{b} \oplus X^{b}$ with the quasi-isogeny $\rho=\rho^{b} \oplus \rho^{b}$ and polarization $\lambda=\lambda^{b} \oplus \lambda^{b}$.

By the functoriality of Rapoport-Zink spaces ([im13, 4.9.6]), we have a commutative diagram of closed immersions

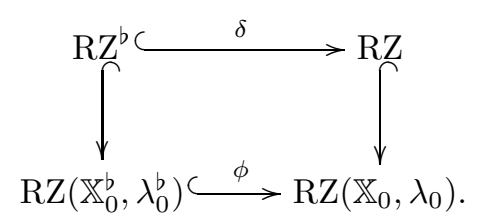

Here the two vertical arrows are induced by the closed immersions $\operatorname{GSpin}\left(V^{b}\right) \hookrightarrow \operatorname{GSp}\left(C\left(V^{b}\right), \psi^{b}\right)$ and $\operatorname{GSpin}(V) \hookrightarrow \operatorname{GSp}(C(V), \psi)(\$ 2.4)$.

Lemma 3.2.1. Diagram (3.2.0.1) is Cartesian, i.e., we have

$$
\delta\left(\mathrm{RZ}^{b}\right)=\phi\left(\mathrm{RZ}\left(\mathbb{X}_{0}^{b}, \lambda_{0}^{b}\right)\right) \cap \mathrm{RZ}
$$

inside $\mathrm{RZ}\left(\mathbb{X}_{0}, \lambda_{0}\right)$.

Proof. By flat descent, to show that the closed formal subschemes on the two sides of (3.2.1.1) agree, it suffices to show that they have the same $k$-points and the same formal completion at every $k$-point (cf. [BP17, 5.2.7]). The claim then follows from the observation that both the $k$-points and the formal completions have purely group theoretic description.

In fact, the $k$-points of $\mathrm{RZ}^{b}=\mathrm{RZ}_{G^{b}}, \mathrm{RZ}\left(\mathbb{X}_{0}^{b}, \lambda_{0}^{b}\right)=\mathrm{RZ}_{H}$ and $\mathrm{RZ}=\mathrm{RZ}_{G}$ have the group theoretic description as the affine Deligne-Lusztig sets (2.11.1.2) associated to the groups $G^{b}=\mathrm{GSpin}\left(V^{b}\right), H=$ $\operatorname{GSp}\left(C\left(V^{b}\right), \psi^{b}\right)$ and $G=\operatorname{GSpin}(V)$ respectively. Since $G^{b}=H \cap G$ inside $\operatorname{GL}(C(V))$, we know that both sides of (3.2.1.1) have the same $k$-points. Fix a $k$-point $x \in \mathrm{RZ}^{b}(k)$, then by [HP15, 3.2.12], $\widehat{\mathrm{RZ}}_{G^{b}, x}$ can be identified with $U_{G^{b}}^{\mu_{x}, \wedge}$, where $\mu_{x}: \mathbb{G}_{m, W} \rightarrow G_{W}^{b}$ gives a filtration that lifts the Hodge filtration for $x$, $U_{G^{b}}^{\mu_{x}} \subseteq G^{b}$ is the unipotent radical of the opposite parabolic group defined by $\mu_{x}([\mathrm{HP} 15,3.1 .6])$ and $U_{G^{b}}^{\mu_{x}, \wedge}$ is its formal completion along its identity section over $W$. Similarly, we can identify $\widehat{\mathrm{RZ}}_{H, x}$ and $\widehat{\mathrm{RZ}}_{G, x}$ as $U_{H}^{\mu_{x}, \wedge}$ and $U_{G}^{\mu_{x}, \wedge}$. Again because $G^{b}=H \cap G$, we know that the formal completions at $x$ of both sides of (3.2.1.1) agree inside $U_{\mathrm{GL}(C(V))}^{\mu_{x}, \wedge}$

Lemma 3.2.2. $\delta\left(\mathrm{RZ}^{\mathrm{b}}\right)=\mathcal{Z}\left(x_{n}\right)$.

Proof. Let $X^{b}$ be the universal $p$-divisible group over $\mathrm{RZ}^{b}$ and $\rho^{b}$ be the universal quasi-isogeny. Then it follows from the commutative diagram (3.2.0.1) that the image of $\left(X^{b}, \rho\right)$ under $\delta$ is given by the $p$-divisible group $\left(X^{b} \oplus X^{b}, \rho^{b} \oplus \rho^{b}\right)$. Since $x_{n}$ has norm 1 , right multiplication by $x_{n}$ swaps the two factors $C\left(V^{b}\right)$ and $C\left(V^{b}\right) x_{n}$. It follows that the quasi-endomorphism

$$
\left(\rho^{b} \oplus \rho^{b}\right) \circ x_{n} \circ\left(\rho^{b} \oplus \rho^{b}\right)^{-1}:\left(X^{b} \oplus X^{b}\right) \rightarrow\left(X^{b} \oplus X^{b}\right)
$$


(uniquely determined by the rigidity of quasi-isogenies) simply swaps the two factors, which is an actual endomorphism (i.e., swapping) of $X^{\mathrm{b}} \oplus X^{\mathrm{b}}$. By Definition 2.14.1 of $\mathcal{Z}\left(x_{n}\right)$, we have $\delta\left(\mathrm{RZ}^{\mathrm{b}}\right) \subseteq \mathcal{Z}\left(x_{n}\right)$.

Conversely, over $\mathcal{Z}\left(x_{n}\right)$ the universal $p$-divisible group $X$ admits an action of $C\left(x_{n}\right)^{\text {op }} \otimes C(V)$, where $C\left(x_{n}\right)$ is the Clifford algebra of the rank one quadratic space $\mathbb{Z}_{p} x_{n}$. Notice

$$
C\left(x_{n}\right)^{\mathrm{op}} \otimes C(V) \cong\left(C\left(x_{n}\right)^{\mathrm{op}} \otimes C\left(x_{n}\right)\right) \oplus\left(C\left(x_{n}\right)^{\mathrm{op}} \otimes C\left(V^{\mathrm{b}}\right)\right) .
$$

It follows that over $\mathcal{Z}\left(x_{n}\right)$ the universal $p$-divisible group $X$ admits an action of $C\left(x_{n}\right)^{\mathrm{op}} \otimes C\left(x_{n}\right)$, which is isomorphic to the matrix algebra $M_{2}\left(\mathbb{Z}_{p}\right)$. The two natural idempotents of $M_{2}\left(\mathbb{Z}_{p}\right)$ then decomposes $X$ as a direct sum of the form $X^{b} \oplus X^{b}$. Hence $\mathcal{Z}\left(x_{n}\right) \subseteq \phi\left(\mathrm{RZ}\left(\mathbb{X}_{0}^{b}, \lambda_{0}^{b}\right)\right) \cap \mathrm{RZ}$. The latter is equal to $\delta\left(\mathrm{RZ} Z^{b}\right)$ by (3.2.1.1) and hence $\mathcal{Z}\left(x_{n}\right) \subseteq \delta\left(\mathrm{RZ}^{\mathrm{b}}\right)$.

Remark 3.2.3. In the following we will only use the inclusion $\delta\left(\mathrm{RZ}^{\mathrm{b}}\right) \subseteq \mathcal{Z}\left(x_{n}\right)$.

\subsection{Arithmetic intersection of GGP cycles.}

Definition 3.3.1. The closed immersion $\delta$ induces a closed immersion of formal schemes

$$
(\mathrm{id}, \delta): \mathrm{RZ}^{\mathrm{b}} \rightarrow \mathrm{RZ}^{\mathrm{b}} \times_{W} \mathrm{RZ} .
$$

Denote by $\Delta$ the image of (id, $\delta$ ), which we call the GGP cycle.

The embedding $V^{b} \hookrightarrow V$ also induces an embedding of quadratic spaces $V_{K}^{b, \Phi} \hookrightarrow V_{K}^{\Phi}$ and hence we can view

$$
J_{b^{\mathrm{b}}}=\operatorname{GSpin}\left(V_{K}^{b, \Phi}\right) \hookrightarrow J_{b}
$$

as an algebraic subgroup over $\mathbb{Q}_{p}$.

For any $g \in J_{b}\left(\mathbb{Q}_{p}\right)$, we obtain a formal subscheme

$$
g \Delta:=(\mathrm{id} \times g) \Delta \subseteq \mathrm{RZ}^{b} \times_{W} \mathrm{RZ},
$$

via the action of $g$ on RZ. Our goal is to compute the arithmetic intersection number

$$
\langle\Delta, g \Delta\rangle,
$$

when $g$ is regular semisimple and minuscule.

Definition 3.3.2. We say $g \in J_{b}\left(\mathbb{Q}_{p}\right)$ is regular semisimple if the $\mathbf{v}(g):=\left(x_{n}, g x_{n}, \ldots, g^{n-1} x_{n}\right)$ forms a $\mathbb{Q}_{p}$-basis of $V_{K}^{\Phi}$. Equivalently, the fundamental matrix $T(g):=T(\mathbf{v}(g))$ is non-singular (Definition 2.14.1). We say $g$ is minuscule if $\mathbf{v}(g)$ is minuscule (Definition 2.14.3).

3.4. Fixed points. Let $g \in J_{b}\left(\mathbb{Q}_{p}\right)$ and let $\mathrm{RZ}^{g} \subseteq \mathrm{RZ}$ be the fixed locus of $g$. Then by definition we have

$$
\Delta \cap g \Delta \cong \delta\left(\mathrm{RZ}^{\mathrm{b}}\right) \cap \mathrm{RZ}^{g} .
$$

Definition 3.4.1. Let $g \in J_{b}\left(\mathbb{Q}_{p}\right)$ be regular semisimple. We define the lattice

$$
L(g):=\mathbb{Z}_{p} x_{n}+\cdots \mathbb{Z}_{p} g^{n-1} x_{n} \subseteq V_{K}^{\Phi} .
$$

Lemma 3.4.2. Inside $\mathrm{RZ}$ both the formal subschemes $\mathrm{RZ}^{g}$ and $\delta\left(\mathrm{RZ}^{\mathrm{b}}\right)$ are stable under $p^{\mathbb{Z}}$. Moreover, under the bijection (2.11.1.1), we have

(1) $p^{\mathbb{Z}} \backslash \delta\left(\mathrm{RZ} Z^{b}(k)\right) \cong\left\{L=L^{b} \oplus W x_{n}: L^{b} \subseteq V_{K}^{b}\right.$ special lattices $\}$.

(2) $p^{\mathbb{Z}} \backslash \delta\left(\mathrm{RZ}^{\mathrm{b}}(k)\right) \cong\left\{L\right.$ special lattices $\left.: x_{n} \in L\right\}$.

(3) $p^{\mathbb{Z}} \backslash \mathrm{RZ} Z^{g}(k) \cong\{L$ special lattices $: g L=L\}$.

(4) $p^{\mathbb{Z}} \backslash\left(\delta\left(\mathrm{RZ}^{\mathrm{b}}(k)\right) \cap \mathrm{RZ}^{g}(k)\right) \cong\left\{L\right.$ special lattices $\left.: g L=L, L \supseteq L(g)_{W}\right\}$. 
Proof. Since $p^{\mathbb{Z}}$ is central in $J_{b}\left(\mathbb{Q}_{p}\right)$, we know $\mathrm{RZ}^{g}$ is stable under $p^{\mathbb{Z}}$. The morphism $\delta: \mathrm{RZ} \mathrm{b}^{b} \rightarrow \mathrm{RZ}$ is equivariant with respect to the natural inclusion $J_{b^{b}}\left(\mathbb{Q}_{p}\right) \rightarrow J_{b}\left(\mathbb{Q}_{p}\right)$, and the morphism $J_{b^{b}} \rightarrow J_{b}$ restricts to the identity between the centers $\mathbb{G}_{m}$ of $J_{b^{b}}$ and of $J_{b}$. It follows that $\delta$ is equivariant for the $p^{\mathbb{Z}}$ action, and so $\delta\left(\mathrm{RZ} Z^{b}\right)$ is stable under $p^{\mathbb{Z}}$. We now prove the statements (1) to (4).

(1) For a point $L^{b} \in p^{\mathbb{Z}} \backslash \mathrm{R} Z^{b}(k)$, we can write $L^{b}=h^{b} \mu^{b}\left(p^{-1}\right) \cdot V_{W}^{b} \subseteq V_{K}^{b}$, for some $h^{b} \in G^{b}(K)$. Then its image under $\delta$ is given by $L=h \mu\left(p^{-1}\right) . V_{W} \subseteq V_{K}$, where $h$ is the image of $h^{\mathrm{b}}$ in $G(K)$. By $V=V^{\mathrm{b}} \oplus \mathbb{Z}_{p} x_{n}$ and the compatibility between $h, \mu$ and $h^{b}, \mu^{b}$, we know that $L=L^{\mathrm{b}} \oplus W x_{n}$.

(2) Suppose $L$ is a special lattice with $x_{n} \in L$. Since $x_{n}$ has norm 1 , we know that $L=L^{\prime} \oplus W x_{n}$ is the direct sum of $W x_{n}$ and its orthogonal complement $L^{\prime}$ in $L$. One can check $L^{\prime} \subseteq V_{K}^{b}$ is also a special lattice. This finishes the proof in view of item (1).

(3) This is clear since $\mathrm{RZ}^{g}(k)$ is the fixed locus of $g$.

(4) For a point $L \in p^{\mathbb{Z}} \backslash\left(\delta\left(\mathrm{RZ}^{\mathrm{b}}(k)\right) \cap \mathrm{RZ}^{g}(k)\right)$, by items (1) (3), we have $L=L^{\mathrm{b}} \oplus W x_{n}$ and $g L=L$. It follows from $x_{n} \in L$ that $g x_{n}, \ldots, g^{n-1} x_{n} \in L$, and so $L \supseteq L(g)_{W}$. Conversely, if a point $L \in \mathrm{RZ}(k)$ satisfies $g L=L$ and $L \supset L(g)_{W}$, then $L \in p^{\mathbb{Z}} \backslash\left(\delta\left(\mathrm{RZ}^{\mathrm{b}}(k)\right) \cap \mathrm{RZ}^{g}(k)\right)$ by items (2) and (3)

Definition 3.4.3. We say a vertex lattice $\Lambda$ is a g-vertex lattice if $g \Lambda=\Lambda$ and $\Lambda \subseteq L(g)^{\vee}$. Denote the set of all $g$-vertex lattices by $\operatorname{VL}(g)$. In general, if a vertex lattice $\Lambda$ satisfies $g \Lambda=\Lambda$, then $g$ induces an action on $\Omega_{0}=\Lambda / \Lambda^{\vee}$, which further induces an action $\bar{g}$ on $\mathrm{RZ}_{\Lambda}^{\text {red }}$ and $\mathrm{BT}_{\Lambda}$. We denote the fixed locus of $\bar{g}$ on $\mathrm{BT}_{\Lambda}$ by $\mathrm{BT}_{\Lambda}^{\bar{g}}$.

Proposition 3.4.4.

$$
p^{\mathbb{Z}} \backslash\left(\delta\left(\mathrm{RZ}^{\mathrm{b}}\right) \cap \mathrm{RZ}^{g}\right)(k)=\coprod_{\Lambda \in \mathrm{VL}(g)} p^{\mathbb{Z}} \backslash \mathrm{BT}_{\Lambda}^{\bar{g}}(k) .
$$

Proof. By Lemma 3.4.2, it suffices to show the $k$-points of the right hand side are in bijection with special lattices $L$ such that $g L=L$ and $L \supseteq L(g)_{W}$. Notice that any special lattice $L$ is self-dual, so the condition $L \supseteq L(g)_{W}$ is equivalent to the condition $L \subseteq L(g)_{W}^{\vee}$. Since $\Lambda(L)_{W}$ is the minimal $\Phi$-invariant lattice containing $L$ (\$2.12), and $L(g)_{W}^{\vee}$ is $\Phi$-invariant, we know that the condition $L \subseteq L(g)_{W}^{\vee}$ is equivalent to the condition $\Lambda(L) \subseteq L(g)^{\vee}$. The result now follows from taking $\bar{g}$-invariants and $g$-invariants of the two sides of the bijection (2.12.1.1).

3.5. Fixed points in a Bruhat-Tits stratum. Let $\Lambda$ be a vertex lattice and $\Omega_{0}=\Lambda / \Lambda^{\vee}$ (2.7). By the isomorphism (2.13.0.2),$p^{\mathbb{Z}} \backslash \mathrm{BT}_{\Lambda}$ is disjoint union of two isomorphic Deligne-Lusztig varieties $X_{B}\left(w^{ \pm}\right)$ associated to the Coxeter elements $w^{ \pm}$for $\mathrm{SO}\left(\Omega_{0}\right)$. Write $X:=X_{B}\left(w^{ \pm}\right)$. To compute $p^{\mathbb{Z}} \backslash \mathrm{BT} T_{\Lambda}^{\bar{g}}$, it suffices to compute the $\bar{g}$-fixed points $X^{\bar{g}}$.

Definition 3.5.1. We say a semisimple element $\bar{g} \in \mathrm{SO}\left(\Omega_{0}\right)$ is regular if $Z^{\circ}(\bar{g})$, the identity component of the centralizer of $\bar{g}$ in $\mathrm{SO}\left(\Omega_{0}\right)$, is a (necessarily maximal) torus.

Proposition 3.5.2. Let $\Lambda$ be a vertex lattice and let $\bar{g} \in \mathrm{SO}\left(\Omega_{0}\right)\left(\mathbb{F}_{p}\right)$.

(1) $X^{\bar{g}}$ is non-empty if and only if $\bar{g}$ is semisimple and contained in a maximal torus of Coxeter type.

(2) $X^{\bar{g}}$ is non-empty and finite if and only if $\bar{g}$ is regular semisimple and contained in a maximal torus of Coxeter type. In this case, the cardinality of $X^{\bar{g}}$ is given by $t_{\Lambda} / 2$.

Remark 3.5.3. Recall that a maximal torus $T^{\prime}$ is of Coxeter type if $T^{\prime}=h T h^{-1}$ for some $h \in \operatorname{SO}\left(\Omega_{0}\right)$ such that $h^{-1} \Phi(h)$ lifts to a Coxeter element $w$ in the Weyl group $W(T)=N(T) / T$. In other words, $T^{\prime}$

\footnotetext{
${ }^{1}$ Note the difference with Definition 3.3 .2 The conflict of the usage of the word "regular" should hopefully not cause confusion.
} 
is conjugate to $T$ over $k$ but its Frobenius structure is given by $w \cdot \Phi$. For the Coxeter element $w=w^{ \pm}$ constructed in 2.13 we know that an element $\left(\lambda_{1}, \ldots, \lambda_{d}, \lambda_{d}^{-1}, \ldots, \lambda_{1}^{-1}\right)$ of $T(k)$ is fixed by $w \cdot \Phi$ if and only if

$$
\left(\lambda_{1}, \lambda_{2}, \ldots, \lambda_{d-1}, \lambda_{d}\right)=\left(\lambda_{d}^{\mp p}, \lambda_{1}^{p} \ldots, \lambda_{d-2}^{p}, \lambda_{d-1}^{ \pm p}\right) .
$$

It follows that a semisimple element $\bar{g} \in \mathrm{SO}\left(\Omega_{0}\right)\left(\mathbb{F}_{p}\right)$ is contained in a maximal torus of Coxeter type if and only if the eigenvalues of $\bar{g}$ on $\Omega_{0} \otimes k$ belong to a single Galois orbit.

Proof. (1) Suppose $X^{\bar{g}}$ is non-empty. Then it is a general fact about Deligne-Lusztig varieties that $\bar{g}$ must be semisimple ([Lus11, 5.9 (a)]). Let $T(w) \subseteq \mathrm{SO}\left(\Omega_{0}\right)$ be a torus of Coxeter type (associated to $w=w^{+}$ or $w^{-}$) and $B(w) \supseteq T(w)$ be a Borel. Assume $\bar{g}$ is semisimple. Then we know from [DL76, Proposition 4.7] that $X^{\bar{g}}$ is a disjoint union of Deligne-Lusztig varieties $X_{T^{\prime} \subseteq B^{\prime}}$ for the group $G^{\prime}=Z^{\circ}(\bar{g})$ and the pairs

$$
\left(T^{\prime}, B^{\prime}\right)=\left(h T(w) h^{-1}, h B(w) h^{-1} \cap G^{\prime}\right),
$$

where $h$ runs over classes $G^{\prime}\left(\mathbb{F}_{p}\right) \backslash \mathrm{SO}\left(\Omega_{0}\right)\left(\mathbb{F}_{p}\right)$ such that $\bar{g} \in h T(w) h^{-1}$. Therefore $X^{\bar{g}}$ is non-empty if and only if there exists $h \in \mathrm{SO}\left(\Omega_{0}\right)\left(\mathbb{F}_{p}\right)$ such that $\bar{g} \in h T(w) h^{-1}$, if and only if $\bar{g}$ is contained in a maximal torus of Coxeter type (as so is $T(w)$ ).

(2) By part (1) we know that $X^{\bar{g}}$ is further finite if and only if all $X_{T^{\prime} \subseteq B^{\prime}}$ are zero dimensional, if and only if all $B^{\prime}=h B h^{-1} \cap G^{\prime}$ are tori. This happens exactly when $G^{\prime}=Z^{\circ}(\bar{g})$ itself is a torus, i.e., when $\bar{g}$ is regular. In this case, $G^{\prime}$ is a maximal torus of Coxeter type in $\mathrm{SO}\left(\Omega_{0}\right)$ and the cardinality of $X^{\bar{g}}$ is equal to the cardinality of $N(T(w))\left(\mathbb{F}_{p}\right) / T(w)\left(\mathbb{F}_{p}\right)$. The latter group is isomorphic to $(N(T(w)) / T(w))^{\Phi}$ by Lang's theorem and hence is isomorphic to the $\Phi$-twisted centralizer of $w$ in the Weyl group $W(T)=$ $N(T) / T$ :

$$
Z_{\Phi}(w):=\{x \in W(T): x w=w \Phi(x)\} .
$$

The cardinality of $Z_{\Phi}(w)$ is known as the Coxeter number of the group $\mathrm{SO}\left(\Omega_{0}\right)$, which is equal to $d=t_{\Lambda} / 2$ since $\mathrm{SO}\left(\Omega_{0}\right)$ is a non-split even orthogonal group ([Lus77, 1.15]).

3.6. Point-counting in the minuscule case. Let $g \in J_{b}\left(\mathbb{Q}_{p}\right)$ be regular semisimple and minuscule. Then $\Omega_{0}(g):=L(g)^{\vee} / L(g)$ is a $\mathbb{F}_{p}$-vector space (see Definition 2.14.3), and hence $L(g)^{\vee}$ is a vertex lattice.

Remark 3.6.1. If $\mathrm{RZ}^{g}$ is non-empty, then $g$ fixes some vertex lattice and so we know that the characteristic polynomial of $g$ has $\mathbb{Z}_{p}$-coefficients. It follows that $L(g)$ is a $g$-stable lattice, from which it also follows easily that $L(g)^{\vee}$ is $g$-stable. Hence by definition $L(g)^{\vee}$ is a $g$-vertex lattice. The induced action of $g$ on $\Omega_{0}(g)$, denoted by $\bar{g} \in \mathrm{SO}\left(\Omega_{0}(g)\right)\left(\mathbb{F}_{p}\right)$, makes $\Omega_{0}(g)$ a $\bar{g}$-cyclic $\mathbb{F}_{p}$-vector space. It follows that the minimal polynomial of $\bar{g}$ is equal to its characteristic polynomial.

From now on we assume $\mathrm{RZ}^{g}$ is non-empty. Let $\bar{g} \in \mathrm{SO}\left(\Omega_{0}(g)\right)\left(\mathbb{F}_{p}\right)$ be as in Remark 3.6.1.

Definition 3.6.2. For any polynomial $R(T)$, we define its reciprocal to be

$$
R^{*}(T):=T^{\operatorname{deg} R(T)} \cdot R(1 / T) .
$$

We say $R(T)$ is self-reciprocal if $R(T)=R^{*}(T)$.

Definition 3.6.3. Let $P(T) \in \mathbb{F}_{p}[T]$ be the characteristic polynomial of $\bar{g} \in \mathrm{SO}\left(\Omega_{0}(g)\right)$. Then $P(T)$ is self-reciprocal. For any monic irreducible factor $Q(T)$ of $P(T)$, we denote by $m(Q(T))$ to be the multiplicity of $Q(T)$ appearing in $P(T)$. 
Theorem 3.6.4. Assume $\mathrm{RZ}^{g}$ is non-empty. Then $p^{\mathbb{Z}} \backslash\left(\delta\left(\mathrm{RZ}^{b}\right) \cap \mathrm{RZ}^{g}\right)(k)$ is non-empty if and only if $P(T)$ has a unique self-reciprocal monic irreducible factor $Q(T)$ such that $m(Q(T))$ is odd. In this case, $p^{\mathbb{Z}} \backslash\left(\delta\left(\mathrm{RZ}^{\mathrm{b}}\right) \cap \mathrm{RZ}^{g}\right)(k)$ is finite and has cardinality

$$
\operatorname{deg} Q(T) \cdot \prod_{R(T)}(1+m(R(T)))
$$

where $R(T)$ runs over all non-self-reciprocal monic irreducible factors of $P(T)$.

Proof. By Proposition 3.4.4, we know that $p^{\mathbb{Z}} \backslash\left(\delta\left(\mathrm{RZ}^{\mathrm{b}}\right) \cap \mathrm{RZ} \mathrm{Z}^{g}\right)(k)$ is non-empty if and only if $p^{\mathbb{Z}} \backslash \mathrm{BT}_{\Lambda}^{g}$ is non-empty for some $\Lambda \in \mathrm{VL}(g)$. For any $\Lambda \in \mathrm{VL}(g)$, by definition we have a chain of inclusions of lattices

$$
L(g) \subseteq \Lambda^{\vee} \subseteq \Lambda \subseteq L(g)^{\vee},
$$

which induces a filtration of $\mathbb{F}_{p}$-vector spaces,

$$
0 \subseteq \Lambda^{\vee} / L(g) \subseteq \Lambda / L(g) \subseteq \Omega_{0}(g) .
$$

It follows that the map $\Lambda \mapsto \Lambda^{\vee} / L(g)$ gives a bijection

$$
\mathrm{VL}(g) \cong\left\{\text { totally isotropic } \bar{g} \text {-invariant subspaces } U \subseteq \Omega_{0}(g)\right\} \text {. }
$$

By the bijection (3.6.4.1), $\mathrm{VL}(g)$ is non-empty if and only if there is a totally isotropic $\bar{g}$-invariant subspace $U$ of $\Omega_{0}(g)$. Such a subspace $U$ induces a filtration

$$
0 \subseteq U \subseteq U^{\perp} \subseteq \Omega_{0}(g)
$$

Since $U$ and $U^{\perp}$ are $\bar{g}$-invariant, we obtain a decomposition of the characteristic polynomial

$$
P(T)=P_{1}(T) Q(T) P_{2}(T)
$$

where $P_{1}(T), Q(T), P_{2}(T)$ are respectively the characteristic polynomials of $\bar{g}$ acting on the associated graded $U, U^{\perp} / U$ and $\Omega_{0}(g) / U^{\perp}$. Notice the non-degenerate quadratic form on $\Omega_{0}(g)$ identifies $\Omega_{0}(g) / U^{\perp}$ with the linear dual of $U$, from which we know that $P_{2}(T)=P_{1}^{*}(T)$. Similarly, we know that $Q(T)=Q^{*}(T)$, i.e., $Q(T)$ is self-reciprocal.

Let $\Lambda=L(g)+U^{\perp}$ be the $g$-vertex lattice corresponding to $U$ under the bijection (3.6.4.1) and let $\Omega_{0}=\Lambda / \Lambda^{\vee}$ and $\bar{g}_{0} \in \mathrm{SO}\left(\Omega_{0}\right)\left(\mathbb{F}_{p}\right)$ be the induced action of $\bar{g}$ on $\Omega_{0}$. By Remark 3.6.1, the minimal polynomial of $\bar{g}$ is equal to its characteristic polynomial $P(T)$. Thus the minimal polynomial of $\bar{g}_{0}$ is equal to its characteristic polynomial $Q(T)$ under the decomposition (3.6.4.3). If $\bar{g}_{0}$ is semisimple, then its eigenvalues are distinct. If $\bar{g}$ is further contained in a torus of Coxeter type, then we know that its eigenvalues belong to a single Galois orbit (Remark 3.5.3), so $Q(T)$ is irreducible. Conversely, if $Q(T)$ is irreducible, then clearly $\bar{g}_{0}$ is semisimple and contained in a torus of Coxeter type. Hence we know that $\bar{g}_{0}$ is semisimple and contained in a torus of Coxeter type if and only if $Q(T)$ is irreducible.

Therefore by Proposition 3.5 .2 (1), $\mathrm{BT}_{\Lambda}^{\bar{g}_{0}}$ is non-empty if and only if $Q(T)$ is irreducible. In this case, $\bar{g}_{0}$ is indeed regular semisimple and the cardinality of $p^{\mathbb{Z}} \backslash \mathrm{BT}_{\Lambda}^{\bar{g}_{0}}$ is equal $2 \cdot \# X^{\bar{g}_{0}}$ (due to two connected components), which is equal to $\operatorname{dim}_{\mathbb{F}_{p}} \Omega_{0}=\operatorname{deg} Q(T)$ by Proposition 3.5 .2 (2).

Since $P_{2}(T)=P_{1}^{*}(T)$, we know the multiplicity of $R(T)$ in $P_{1}(T) P_{2}(T)$ is even for any self-reciprocal factor $R(T)$. Hence $Q(T)$ is the unique self-reciprocal monic irreducible factor of $P(T)$ such that $m(Q(T))$ is odd. Finally, the factorizations (3.6.4.3) with $P_{2}(T)=P_{1}^{*}(T)$ corresponds bijectively to the filtrations (3.6.4.2). The proof is now finished by noticing that the number of such factorizations is exactly given by

$$
\prod_{R(T)}(1+m(R(T)))
$$

where $R(T)$ runs over all monic irreducible factors of $P(T)$ such that $R(T) \neq R^{*}(T)$. 


\section{The REDUCEDNESS OF MINUSCUlE SPECIAL CYCLES}

\subsection{The analogue of a result of Madapusi Pera on special cycles.}

Definition 4.1.1. Let $\mathcal{O}$ be an arbitrary $\mathbb{Z}[1 / 2]$-algebra. Assume $\mathcal{O}$ is local. Let $\mathbf{L}$ be a finite free $\mathcal{O}$-module equipped with the structure of a self-dual quadratic space over $\mathcal{O}$. By an isotropic line in $\mathbf{L}$ we mean a direct summand of rank one on which the quadratic form is zero.

We start with a general lemma on Clifford algebras.

Lemma 4.1.2. Let $\mathcal{O}$ and $\mathbf{L}$ be as in Definition 4.1.1. Let $C(\mathbf{L})$ be the associated Clifford algebra. Let $\xi \in \mathbf{L}$ be an $\mathcal{O}$-generator of an isotropic line. Let $\operatorname{ker}(\xi)$ be the kernel of the endomorphism of $C(\mathbf{L})$ given by left multiplication by $\xi$. Then for any $v \in \mathbf{L}$, left multiplication by $v$ preserves $\operatorname{ker}(\xi)$ if and only if $v$ is orthogonal to $\xi$.

Proof. Assume $v$ is orthogonal to $\xi$. Then $v \xi=-\xi v$, so $v$ preserves $\operatorname{ker}(\xi)$.

Conversely, assume $v$ preserves $\operatorname{ker}(\xi)$. Write $q$ for the quadratic form and $\langle$,$\rangle the corresponding bilinear$ pairing. Since $\mathcal{O} \xi$ is a direct summand of $\mathbf{L}$, there exists an $\mathcal{O}$-module homomorphism $\mathbf{L} \rightarrow \mathcal{O}$ sending $\xi$ to 1 . Since $\mathbf{L}$ is self-dual, we know that there exists $\zeta \in \mathbf{L}$ representing such a homomorphism. Namely we have

$$
\langle\zeta, \xi\rangle=1
$$

It immediately follows that we have an $\mathcal{O}$-module direct sum $\mathbf{L}=\xi^{\perp} \oplus \mathcal{O} \zeta$. Replacing $\zeta$ by $\zeta-\frac{q(\zeta)}{2} \xi$, we may arrange that $\zeta$ is isotropic. We have

$$
q(\zeta+\xi)=2\langle\zeta, \xi\rangle=2,
$$

and in $C(\mathbf{L})$ we have

$$
q(\zeta+\xi)=\zeta \xi+\xi \zeta
$$

Hence in $C(\mathbf{L})$ we have

$$
\xi \zeta+\zeta \xi=2
$$

Write

$$
v=v_{1}+\lambda \zeta,
$$

with $v_{1} \in \xi^{\perp}$ and $\lambda \in \mathcal{O}$. By the first part of the proof we know that $v_{1}$ preserves $\operatorname{ker}(\xi)$. Therefore $\lambda \zeta$ preserves $\operatorname{ker}(\xi)$. Note that $\xi \in \operatorname{ker}(\xi)$ as $\xi$ is isotropic. It follows that, in $C(\mathbf{L})$,

$$
0 \stackrel{\lambda \zeta \text { preserves } \operatorname{ker}(\xi)}{=} \xi(\lambda \zeta) \xi \stackrel{4.1 .2 .1}{=} \lambda(2-\zeta \xi) \xi \stackrel{\xi \text { isotropic }}{=} 2 \lambda \xi \text {. }
$$

This is possible only when $\lambda=0$, and hence we have $v=v_{1} \in \xi^{\perp}$.

The next result is a Rapoport-Zink space analogue of [MP16, Proposition 5.16] which is in the context of special cycles on GSpin Shimura varieties. We only state a weaker analogue as it is sufficient for our need. The proof builds on loc. cit. too. We first introduce some definitions.

Definition 4.1.3. Denote by $y_{00}$ the distinguished $k$-point of RZ corresponding to $\mathbb{X}_{0}$ and the identity quasi-isogeny. Let $y_{0} \in \mathrm{RZ}(k)$ be an arbitrary element. Let $L$ be the special lattice corresponding to $y_{0}$ under (2.11.1.1). When $y_{0}=y_{00}$, we have $\Phi L=V_{W}$ (cf. the discussion below (2.11.1.1)). In this case define $\operatorname{Fil}^{1}(\Phi L)_{k}$ to be the one-dimensional subspace of $V_{k}$ defined by the cocharacter $\mu$ of $G_{W}$ and the representation $G_{k} \rightarrow \mathrm{GL}\left(V_{k}\right)$. For general $y_{0}$, let $g \in X_{G, b, \mu^{\sigma}}(k)$ be associated to $y_{0}$. Then $\Phi L=g V_{W}$ and $g$ induces a map $V_{k} \rightarrow(\Phi L)_{k}$ (cf. loc. cit.). Define Fil ${ }^{1}(\Phi L)_{k}$ to be the image of Fil ${ }^{1} V_{k}$ under the last map. 
Remark 4.1.4. By our explicit choice of $\mu$ in 2.3 the submodule $\mathbb{Z}_{p} x_{2}$ in $V$ is of weight 1 with respect to $\mu$, and $\oplus_{1 \leq i \leq n, i \neq 2} \mathbb{Z}_{p} x_{i}$ is of weight 0 with respect to $\mu$, so Fil $^{1} V_{k}=k x_{2}$.

Remark 4.1.5. Fil $^{1}(\Phi L)_{k}$ is the orthogonal complement in $(\Phi L)_{k}$ of $(L \cap \Phi L)_{k}$. However we will not need this description in the sequel.

Definition 4.1.6. Let $\mathscr{C}$ be the category defined as follows:

- Objects in $\mathscr{C}$ are triples $(\mathcal{O}, \mathcal{O} \rightarrow k, \delta)$, where $\mathcal{O}$ is a local artinian $W$-algebra, $\mathcal{O} \rightarrow k$ is a $W$-algebra map, and $\delta$ is a nilpotent divided power structure on $\operatorname{ker}(\mathcal{O} \rightarrow k)$.

- Morphisms in $\mathscr{C}$ are $W$-algebra maps that are compatible with the structure maps to $k$ and the divided power structures.

In the following we will abuse notation to write $\mathcal{O}$ for an object in $\mathscr{C}$.

Let $y_{0} \in \mathrm{RZ}(k)$ be an arbitrary element. Let $\mathbf{v}$ be as in Definition 2.14.1 such that the special cycle $\mathcal{Z}:=\mathcal{Z}(\mathbf{v})$ contains $y_{0}$. In particular $\mathbf{v} \subset L \cap \Phi L$ by Remark 2.14.2. Let $\widehat{\mathrm{RZ}}_{y_{0}}$ and $\widehat{\mathcal{Z}}_{y_{0}}$ be the formal completions of $\mathrm{RZ}$ and $\mathcal{Z}$ at $y_{0}$ respectively.

Theorem 4.1.7. For any $\mathcal{O} \in \mathscr{C}$ there is a bijection

$$
f_{\mathcal{O}}: \widehat{\mathrm{RZ}}_{y_{0}}(\mathcal{O}) \stackrel{\sim}{\longrightarrow}\left\{\text { isotropic lines in }(\Phi L)_{\mathcal{O}}:=\Phi L \otimes_{W} \mathcal{O} \text { lifting } \operatorname{Fil}^{1}(\Phi L)_{k}\right\}
$$

such that the following properties hold. Here we equip $(\Phi L)_{\mathcal{O}}$ with the $\mathcal{O}$-bilinear form obtained by extension of scalars of the $W$-bilinear form on $\Phi L$.

(1) $f_{\mathcal{O}}$ is functorial in $\mathcal{O} \in \mathscr{C}$ in the following sense. Let $\mathcal{O}^{\prime} \in \mathscr{C}$ be another object of $\mathscr{C}$ and let $\phi: \mathcal{O} \rightarrow \mathcal{O}^{\prime}$ be a morphism in $\mathscr{C}$. Then we have a commuting diagram.

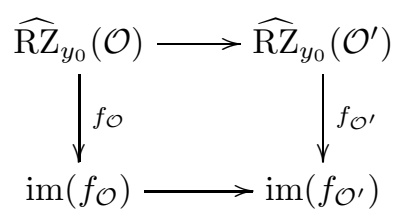

Here the top horizontal map is the natural map induced by $\phi$, and the bottom horizontal map is given by base change along $\phi$.

(2) $f_{\mathcal{O}}$ restricts to a bijection

$$
\begin{aligned}
& \qquad f_{\mathcal{O}, \mathbf{v}}: \widehat{\mathcal{Z}}_{y_{0}}(\mathcal{O}) \stackrel{\sim}{\longrightarrow} \\
& \left\{\text { isotropic lines in }(\Phi L)_{\mathcal{O}} \text { lifting } \mathrm{Fil}^{1}(\Phi L)_{k} \text { and orthogonal to the image of } \mathbf{v} \text { in }(\Phi L)_{\mathcal{O}}\right\}
\end{aligned}
$$

Proof. The existence and construction of the bijection $f_{\mathcal{O}}$ and the property (1) are consequences of [MP16, Proposition 5.16] and the global construction of RZ in [HP15] using the integral model of the GSpin Shimura variety. We explain this more precisely below.

Consider

$$
\mathscr{S}=\mathscr{S}_{U_{p} U^{p}}
$$

the canonical integral model over $\mathbb{Z}_{(p)}$ of the Shimura variety associated to the GSpin Shimura datum associated to a quadratic space $V_{\mathbb{Q}}$ over $\mathbb{Q}$, at a suitable level $U^{p}$ away from $p$ and a hyperspecial level $U_{p}$ at $p$. See [HP15, §7] or [MP16] for more details on this concept. By [HP15, 7.2.3], we may assume that the following package of data:

- the Shimura datum associated to $V_{\mathbb{Q}}$,

- the Kuga-Satake Hodge embedding (cf. [HP15, 4.14]) of the Shimura datum into a GSp Shimura datum, 
- the chosen hyperspecial level at $p$,

- an element $x_{00} \in \lim _{\longleftarrow} \mathscr{S}_{U^{p} U_{p}}(k)$,

induces, in the fashion of [HP15, 3.1.4], the local unramified Shimura-Hodge datum that we used to define RZ. Let $\widehat{\mathscr{S}}$ be the formal scheme over $\mathbb{Z}_{p}$ obtained from $p$-adic completion of $\mathscr{S}$, and let $\widehat{\mathscr{S}} W$ be the base change to $W$ of $\widehat{\mathscr{S}}$. Then as in [HP15, 3.2.14], we have a morphism of formal schemes over $W$ :

$$
\Theta: R Z \rightarrow \widehat{\mathscr{S}}_{W} .
$$

We know that $\Theta$ maps $y_{00}$ to the $k$-point of $\widehat{\mathscr{S}_{W}}$ induced by $x_{00}$. Moreover, let

$$
x_{0}:=\Theta\left(y_{0}\right) \in \widehat{\mathscr{S}}_{W}(k)=\mathscr{S}(k)
$$

and let $\widehat{U}$ be the formal completion of $\mathscr{S}$ at $x_{0}$ (or, what amounts to the same thing, the formal completion of $\widehat{\mathscr{S}_{W}}$ at $x_{0}$ ). By the construction of RZ in [HP15, §3], we know that $\Theta$ induces an isomorphism $\widehat{\mathrm{RZ}}_{y_{0}} \stackrel{\sim}{\longrightarrow} \widehat{U}$.

In [MP16], two crystals $\mathbf{H}_{\text {cris }}, \mathbf{L}_{\text {cris }}$ are constructed on $\mathscr{S}_{k}$. (In fact [MP16] works over $\mathbb{F}_{p}$, but we always base change from $\mathbb{F}_{p}$ to $k$.) Here $\mathbf{H}_{\text {cris }}$ is by definition the first relative crystalline cohomology of the KugaSatake abelian scheme over $\mathscr{S}_{k}$ in the sense of loc. cit 2 The specialization of $\mathbf{H}_{\text {cris }}$ over $\operatorname{Spec} k$ via $x_{00}$ is identified with the Dieudonné module $C_{W}$, which is the covariant Diedonné module of the $p$-divisible group $\mathbb{X}_{0}$ considered in this article (and [HP15]) and the contravariant Diedonné module of the Kuga-Satake abelian variety at $x_{00}$ considered in [MP16] 3 Moreover, the embedding $V \hookrightarrow \operatorname{End}_{\mathbb{Z}_{p}}(C)$ has a cristalline realization, which is a sub-crystal $\mathbf{L}_{\text {cris }}$ of $\operatorname{End}\left(\mathbf{H}_{\text {cris }}\right)$. For details see [MP16, §4]. Among others, $\mathbf{L}_{\text {cris }}$ has the following structures:

- Its specialization $\mathbf{L}_{\text {cris }, x_{0}}$ to any $x_{0} \in \mathscr{S}(k)$, viewed as a $W$-module, has the structure of a $W$-quadratic space.

- $\mathbf{L}_{\text {cris }, x_{0}} \otimes_{W} k$ contains a canonical isotropic line $\operatorname{Fil}^{1}\left(\mathbf{L}_{\mathrm{cris}, x_{0}} \otimes_{W} k\right)$.

By the definition of $\Theta$ and the definition of the parametrization of $\mathrm{RZ}(k)$ by the affine Deligne-Lusztig set (cf. [HP15, §2.4]), we know that when $y_{0} \in \mathrm{RZ}(k)$ corresponds to the special lattice $L$ under (2.11.1.1), the following statements are true:

(a) There is an isomorphism of Dieudonné modules $(g C)_{W} \stackrel{\sim}{\longrightarrow} \mathbf{H}_{\text {cris }, x_{0}}$.

(b) There is a $W$-linear isometry $(\Phi L)_{W} \stackrel{\sim}{\longrightarrow} \mathbf{L}_{\text {cris }, x_{0}}$ under which $\operatorname{Fil}^{1}(\Phi L)_{k}$ is identified with

$$
\operatorname{Fil}^{1}\left(\mathbf{L}_{\mathrm{cris}, x_{0}} \otimes_{W} k\right) .
$$

(c) We have a commutative diagram:

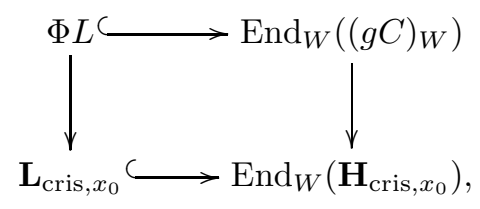

where

- the right vertical map is induced by the map in (a)

- the left vertical map is the map in (b).

- the bottom horizontal map arises from the fact that $\mathbf{L}_{\text {cris }}$ is a sub-crystal of $\operatorname{End}\left(\mathbf{H}_{\text {cris }}\right)$.

\footnotetext{
${ }^{2}$ See footnote 3

${ }^{3}$ Due to different conventions, the Kuga-Satake abelian scheme (and $p$-divisible group) considered by Madapusi Pera in MP16 is different from that considered by Howard-Pappas in HP15. In fact they are dual to each other.
} 
In the rest of the proof we make the identifications in (a) and (b) above and omit them from the notation. Abbreviate $\mathbf{H}:=\mathbf{H}_{\mathrm{cris}, x_{0}}$ and $\mathbf{L}:=\mathbf{L}_{\mathrm{cris}, x_{0}}$.

Now in [MP16, Proposition 5.16] Madapusi Pera constructs a bijection

$$
\widehat{U}(\mathcal{O}) \stackrel{\sim}{\longrightarrow}\left\{\text { isotropic lines in } \mathbf{L}_{\mathcal{O}}:=\mathbf{L} \otimes_{W} \mathcal{O} \text { lifting } \text { Fil }^{1} \mathbf{L}_{k}\right\} .
$$

Moreover by the construction given in loc. cit. the above bijection is functorial in $\mathcal{O} \in \mathscr{C}$. We define $f_{\mathcal{O}}$ as the above bijection precomposed with the isomorphism $\Theta: \widehat{\mathrm{RZ}}_{y_{0}} \stackrel{\sim}{\longrightarrow} \widehat{U}$.

It remains to prove property (2). Note that $\mathbf{H}=g C_{W}$ is the covariant Dieudonné module of the $p$-divisible group $X_{y_{0}}$ over $k$ determined by $y_{0} \in \mathrm{RZ}(k)$. Given $y \in \widehat{\mathrm{RZ}}_{y_{0}}(\mathcal{O})$ lifting $y_{0}$, by Grothendieck-Messing theory (for covariant Dieudonné modules) we know that $y \in \widehat{\mathcal{Z}}_{y_{0}}$ if and only if the image of $\mathbf{v}$ in $\operatorname{End}_{\mathcal{O}}\left(\mathbf{H}_{\mathcal{O}}\right)$ stabilizes Fil $^{1} \mathbf{H}_{\mathcal{O}} \subset \mathbf{H}_{\mathcal{O}}$, where Fil ${ }^{1} \mathbf{H}_{\mathcal{O}}$ is the Hodge filtration corresponding to the deformation from $k$ to $\mathcal{O}$ of the $X_{y_{0}}$ determined by $y$. Now, as is stated in the proof of [MP16, Proposition 5.16], we know that Fil ${ }^{1} \mathbf{H}_{\mathcal{O}}$ is the kernel in $\mathbf{H}_{\mathcal{O}}$ of any $\mathcal{O}$-generator $\xi$ of the isotropic line $f_{\mathcal{O}}(y)$. Here $\xi \in \mathbf{L}_{\mathcal{O}}$ is viewed as an element of $\operatorname{End}_{\mathcal{O}}\left(\mathbf{H}_{\mathcal{O}}\right)$. By Lemma 4.1.2, $\mathbf{v}$ preserves $\mathrm{Fil}^{1} \mathbf{H}_{\mathcal{O}}=\operatorname{ker} \xi$ if and only if $\mathbf{v}$ is orthogonal to $\xi$ (inside $\mathbf{L}_{\mathcal{O}}$ ). Thus $y \in \widehat{\mathcal{Z}}_{y_{0}}$ if and only if $f_{\mathcal{O}}(y)$ is orthogonal to the image of $\mathbf{v}$ in $\mathbf{L}_{\mathcal{O}}=(\Phi L)_{\mathcal{O}}$.

Remark 4.1.8. Consider the bijection $f_{\mathcal{O}, \mathbf{v}}$ for $\mathcal{O}=k$. Since the source of this bijection is non-empty, it follows that $\operatorname{Fil}^{1}(\Phi L)_{k}$ is orthogonal to the image of $\mathbf{v}$ in $(\Phi L)_{k}$. This observation also follows from the Remark 4.1.5 as $\mathbf{v} \subset L \cap \Phi L$.

\subsection{Reducedness of minuscule special cycles.}

Proposition 4.2.1. Let $\Lambda$ be a $\mathbb{Z}_{p}$-lattice in $V_{K}^{\Phi}$ with $p^{i} \Lambda \subset \Lambda^{\vee} \subset \Lambda$ for some $i \in \mathbb{Z}_{\geq 1}$. (Equivalently, $\Lambda^{\vee}$ has invariant $\left(r_{1}, \cdots, r_{n}\right)$ such that $i \geq r_{1} \geq r_{2} \geq \cdots \geq r_{n} \geq 0$.) Then the special cycle $\mathcal{Z}\left(\Lambda^{\vee}\right)$ defined by $\Lambda^{\vee}$ has no $\left(W / p^{i+1}\right)$-points. In particular, taking $i=1$ we see that $\mathrm{RZ}_{\Lambda}\left(W / p^{2}\right)=\varnothing$ for any vertex lattice $\Lambda$, or equivalently $\mathcal{Z}(\mathbf{v})\left(W / p^{2}\right)=\varnothing$ for any minuscule $\mathbf{v}$.

Proof. Suppose there exists $x \in \mathcal{Z}\left(\Lambda^{\vee}\right)\left(W / p^{i+1}\right)$. Let $x_{0} \in \mathcal{Z}\left(\Lambda^{\vee}\right)(k)$ be induced by $x$ under the reduction map $W / p^{i+1} \rightarrow W / p=k$. Under (2.11.1.1) $x_{0}$ determines a special lattice $L$. By Remark 2.14.2, $\Lambda_{W}^{\vee} \subset$ $L \cap \Phi L$. Note that $W / p^{i+1} \rightarrow k$ is a surjection whose kernel admits nilpotent divided powers. By Theorem 4.1.7, the existence of the lift $x$ of $x_{0}$ implies that there exists an isotropic line $\mathcal{L}$ (over $\left.W / p^{i+1}\right)$ in $(\Phi L)_{W / p^{i+1}}$ lifting $\operatorname{Fil}^{1}(\Phi L)_{k}$ and such that $\mathcal{L}$ is orthogonal to the image of $\Lambda^{\vee}$ in $(\Phi L)_{W / p^{i+1}}$. Let $l \in \Phi L$ be a lift of a generator of $\mathcal{L}$. Then $\langle l, \lambda\rangle \in p^{i+1} W$ for all $\lambda \in \Lambda^{\vee}$. It follows that $p^{-(i+1)} l \in \Lambda_{W}$. Hence $p^{-1} l \in p^{i} \Lambda_{W} \subset\left(\Lambda^{\vee}\right)_{W} \subset \Phi L$, i.e. $l \in p \Phi L$. This contradicts with the fact that $\mathcal{L}$ maps to a non-zero line in $(\Phi L)_{k}$.

4.2.2. Let $u \in V_{K}^{\Phi}-\{0\}$. Suppose $x_{0} \in \mathcal{Z}(u)(k)$. Let $T=k[\epsilon] / \epsilon^{2}$ be the ring of dual numbers over $k$. We equip $T$ with the map $T \rightarrow k, \epsilon \mapsto 0$, which has its kernel $(\epsilon)$ admitting nilpotent divided powers (in a unique way). Thus Theorem 4.1 .7 can be applied to $\mathcal{O}=T$.

Let $\mathcal{T}_{x_{0}} \mathrm{RZ}_{k}$ and $\mathcal{T}_{x_{0}} \mathcal{Z}(u)_{k}$ be the tangent spaces at $x_{0}$ to $\mathrm{RZ}_{k}=\mathrm{RZ} \times_{\text {Spf } W} \operatorname{Spec} k$ and to $\mathcal{Z}(u)_{k}=$ $\mathcal{Z}(u) \times_{\text {Spf } W}$ Spec $k$ respectively. We will always take the point of view that $\mathcal{T}_{x_{0}} \mathrm{RZ}_{k}$ is the preimage of $\left\{x_{0}\right\}$ under the reduction map $\mathrm{RZ}(T) \rightarrow \mathrm{RZ}(k)$. Similarly for $\mathcal{T}_{x_{0}} \mathcal{Z}(u)_{k}$. We compute $\mathcal{T}_{x_{0}} \mathrm{RZ}_{k}$ and $\mathcal{T}_{x_{0}} \mathcal{Z}(u)_{k}$ explicitly in the following. The result is given in Corollary 4.2 .7 .

\footnotetext{
${ }^{4}$ Madapusi Pera defines $\mathrm{Fil}^{1} \mathbf{H}_{\mathcal{O}}$ using the contravariant Grothendieck-Messing theory of the $p$-divisible group of the KugaSatake abelian scheme in his sense, which is the same as the covariant Grothendieck-Messing theory of the $p$-divisible group over $\widehat{U}$ transported via $\Theta$ from the universal $p$-divisible group over RZ in the sense of Howard-Pappas.
} 
Let $L$ be the special lattice associated to $x_{0}$ under (2.11.1.1). Since $x_{0} \in \mathcal{Z}(u)(k)$, we have $u \in L \cap \Phi L$ by Remark 2.14.2 Let $\bar{u}$ be the image of $u$ in $(\Phi L)_{k}$. Let Fil $^{1}(\Phi L)_{k}$ be as in Definition 4.1.3. By Remark 4.1 .8 we know that $\bar{u}$ is orthogonal to $\operatorname{Fil}^{1}(\Phi L)_{k}$.

Define $\mathscr{D}$ to be the set of isotropic lines in $(\Phi L)_{T}$ lifting $\operatorname{Fil}^{1}(\Phi L)_{k}$. Define $\mathscr{D}_{u}$ to be the subset of $\mathscr{D}$ consisting of lines which are in addition orthogonal to the image of $u$ in $(\Phi L)_{T}$. Let

$$
\mathscr{G}=f_{T}: \mathcal{T}_{x_{0}} \mathrm{RZ}_{k} \stackrel{\sim}{\longrightarrow} \mathscr{D} .
$$

be the bijection given in Theorem 4.1.7. By the same theorem it restrict to a bijection

$$
\mathcal{T}_{x_{0}} \mathcal{Z}(u)_{k} \stackrel{\sim}{\longrightarrow} \mathscr{D}_{u} .
$$

Definition 4.2.3. We identify $(\Phi L)_{T}$ with $(\Phi L)_{k} \otimes_{k} T$. Fix a $k$-generator $v_{0}$ of $\operatorname{Fil}^{1}(\Phi L)_{k}$. Define a map

$$
\begin{gathered}
\tilde{\mathscr{F}}:(\Phi L)_{k} \rightarrow\left\{T \text {-submodules of }(\Phi L)_{T}\right\} \\
w \mapsto \operatorname{span}_{T}\left\{v_{0} \otimes_{k} 1+w \otimes_{k} \epsilon\right\} .
\end{gathered}
$$

Lemma 4.2.4. $\tilde{\mathscr{F}}$ factors through $(\Phi L)_{k} / \mathrm{Fil}^{1}(\Phi L)_{k}$, and its image consists of $T$-module direct summands of $(\Phi L)_{T}$ of rank one.

Proof. For any $\lambda \in k$, we have

$$
v_{0} \otimes 1+\left(w+\lambda v_{0}\right) \otimes \epsilon=(1+\lambda \epsilon)\left(v_{0} \otimes 1+w \otimes \epsilon\right),
$$

and $1+\lambda \epsilon \in T^{\times}$. Hence $\tilde{\mathscr{F}}$ factors through $(\Phi L)_{k} / \operatorname{Fil}^{1}(\Phi L)_{k}$. For any $w \in(\Phi L)_{k}$, we know that $\tilde{\mathscr{F}}(w)$ is a free module of rank one by definition. It remains to show that $\tilde{\mathscr{F}}(w)$ is a direct summand of $(\Phi L)_{T}$. Let $A$ be a $k$-vector space complement of $\mathrm{Fil}^{1}(\Phi L)_{k}$ inside $(\Phi L)_{k}$. We easily check that the following $T$-submodule of $(\Phi L)_{T}$ is a $T$-module complement of $\tilde{\mathscr{F}}(w)$ :

$$
\operatorname{span}_{T}\left\{v^{\prime} \otimes 1+w \otimes \epsilon \mid v^{\prime} \in A\right\} .
$$

Corollary 4.2.5. The map $\tilde{\mathscr{F}}$ induces a bijection of sets:

$$
\mathscr{F}:\left(\mathrm{Fil}^{1}(\Phi L)_{k}\right)^{\perp} / \mathrm{Fil}^{1}(\Phi L)_{k} \stackrel{\sim}{\longrightarrow} \mathscr{D} .
$$

Moreover, $\mathscr{F}$ restricts to a bijection

$$
\left\{\bar{u}, \operatorname{Fil}^{1}(\Phi L)_{k}\right\}^{\perp} / \operatorname{Fil}^{1}(\Phi L)_{k} \stackrel{\sim}{\longrightarrow} \mathscr{D}_{u} .
$$

Proof. Since $\left\langle v_{0}, v_{0}\right\rangle=0 \in k$, the condition that $\tilde{\mathscr{F}}(w)$ is isotropic is equivalent to $\left\langle w, v_{0}\right\rangle=0 \in k$. Since $v_{0}$ is orthogonal to $\bar{u}$, the condition that $\tilde{\mathscr{F}}(w)$ is orthogonal to the image of $u$ in $(\Phi L)_{T}$ is equivalent to $\langle w, \bar{u}\rangle=0 \in k$.

Lemma 4.2.6. Let $\mathscr{G}$ be as in 4.2.2.1) and let $\mathscr{F}$ be as in Corollary 4.2.5. The map

$$
\mathscr{G}^{-1} \circ \mathscr{F}:\left(\operatorname{Fil}^{1}(\Phi L)_{k}\right)^{\perp} / \mathrm{Fil}^{1}(\Phi L)_{k} \rightarrow \mathcal{T}_{x_{0}} \mathrm{RZ}_{k}
$$

is $k$-linear.

Proof. The proof is a routine check, using the functorial property stated in Theorem 4.1.7.

We first recall the $k$-vector space structure on $\mathcal{T}_{x_{0}} \mathrm{RZ}_{k}$, from the point of view that $\mathcal{T}_{x_{0}} \mathrm{RZ} Z_{k}$ is the preimage of $\left\{x_{0}\right\}$ under the map $\mathrm{RZ}(T) \rightarrow \mathrm{RZ}(k)$. 
Scalar multiplication: Given a tangent vector $v \in \mathcal{T}_{x_{0}} \mathrm{RZ}_{k}$ corresponding to $v_{T} \in \mathrm{RZ}(T)$ and given a scalar $\lambda \in k$, the tangent vector $\lambda v$ corresponds to the following element $(\lambda v)_{T}$ of $\operatorname{RZ}(T)$ : the image of $v_{T}$ under $\mathrm{RZ}(T) \stackrel{T \rightarrow T, \epsilon \mapsto \lambda \epsilon}{\longrightarrow} \mathrm{RZ}(T)$. We see that $(\lambda v)_{T}$ is indeed a preimage of $x_{0}$.

Addition: Let $v_{1}, v_{2} \in \mathcal{T}_{x_{0}} \mathrm{RZ}_{k}$ be two tangent vectors. Let $T_{i}=k\left[\epsilon_{i}\right] / \epsilon_{i}^{2}, i=1,2$ be two copies of $T$. We represent $v_{i}$ as an element $\left(v_{i}\right)_{T_{i}}$ in $\mathrm{RZ}\left(T_{i}\right)$ that reduces to $x_{0} \in \mathrm{RZ}(k)$, for $i=1,2$. Let $\tilde{T}$ be the fiber product of $T_{1}$ and $T_{2}$ over $k$, in the category of $k$-algebras. Namely, $\tilde{T}=k\left[\epsilon_{1}, \epsilon_{2}\right] /\left(\epsilon_{1}, \epsilon_{2}\right)^{2}$. Let $\delta$ be the $k$-algebra map

$$
\delta: \tilde{T} \rightarrow T, \epsilon_{1} \mapsto \epsilon, \epsilon_{2} \mapsto \epsilon .
$$

By the fact that $\tilde{T}$ is the fiber product of $T_{1}$ and $T_{2}$, there is a canonical bijection

$$
\mathrm{RZ}\left(T_{1}\right) \times \mathrm{RZ}\left(T_{2}\right) \stackrel{\sim}{\longrightarrow} \mathrm{RZ}(\tilde{T}) .
$$

Denote by $v_{1} \tilde{+} v_{2}$ the image of $\left(\left(v_{1}\right)_{T_{1}},\left(v_{2}\right)_{T_{2}}\right)$ in $\mathrm{RZ}(\tilde{T})$ under the above bijection. Then the tangent vector $v_{1}+v_{2}$ corresponds to the following element $\left(v_{1}+v_{2}\right)_{T}$ of $\mathrm{RZ}(T)$ : the image of $v_{1} \tilde{+} v_{2}$ under $\delta_{*}: \operatorname{RZ}(\tilde{T}) \rightarrow$ $\mathrm{RZ}(T)$. This last element is indeed a preimage of $x_{0}$.

We now check that $\mathscr{G}^{-1} \circ \mathscr{F}$ is $k$-linear. We first check the compatibility with scalar multiplication. For any $\lambda \in k$ and $w \in\left(\operatorname{Fil}^{1}(\Phi L)_{k}\right)^{\perp}$, we have $\tilde{\mathscr{F}}(w)=\operatorname{span}_{T}\left\{v_{0} \otimes 1+w \otimes_{k} \epsilon\right\}$ and $\tilde{\mathscr{F}}(\lambda w)=\operatorname{span}_{T}\left\{v_{0} \otimes 1+\lambda w \otimes_{k} \epsilon\right\}$. Let $m_{\lambda}$ denote the map $T \rightarrow T, \epsilon \mapsto \lambda \epsilon$. Then we have $\tilde{\mathscr{F}}(w) \otimes_{T, m_{\lambda}} T=\tilde{\mathscr{F}}(\lambda w)$ as submodules of $(\Phi L)_{T}$. By the functoriality in $\mathcal{O}$ stated in Theorem 4.1.7, we know that for all $d \in \mathscr{D}$, the element $\mathscr{G}^{-1}\left(d \otimes_{T, m_{\lambda}} T\right) \in \mathrm{RZ}(T)$ is equal to the image of $\mathscr{G}^{-1}(d)$ under $\mathrm{RZ}(T) \stackrel{\left(m_{\lambda}\right)_{*}}{\longrightarrow} \mathrm{RZ}(T)$. It follows that $\left(\mathscr{G}^{-1} \circ \mathscr{F}\right)(\lambda w)$ is equal to $\lambda$ times the tangent vector $\left(\mathscr{G}^{-1} \circ \mathscr{F}\right)(w)$.

We are left to check the additivity of $\mathscr{G}^{-1} \circ \mathscr{F}$. Let $w_{1}, w_{2} \in\left(\mathrm{Fil}^{1}(\Phi L)_{k}\right)^{\perp}$. Let $\mathscr{D}_{i}, \mathscr{F}_{i}, \mathscr{G}_{i}$ be the analogues of $\mathscr{D}, \mathscr{F}, \mathscr{G}$ respectively with $T$ replaced by $T_{i}$, for $i=1,2$. Also let $f_{\tilde{T}}$ be as in Theorem 4.1.7 (with $\mathcal{O}=\tilde{T}$, where $\operatorname{ker}(\tilde{T} \rightarrow k)$ is equipped with the unique nilpotent divided power structure.) Let $d_{i}:=\mathscr{F}_{i}\left(w_{i}\right), i=1,2$. Then $d_{i}=\operatorname{span}_{T_{i}}\left(v_{0} \otimes 1+w_{i} \otimes \epsilon_{i}\right)$. We easily see that the assertion $\left(\mathscr{G}^{-1} \circ \mathscr{F}\right)\left(w_{1}+w_{2}\right)=\left(\mathscr{G}^{-1} \circ \mathscr{F}\right)\left(w_{1}\right)+$ $\left(\mathscr{G}^{-1} \circ \mathscr{F}\right)\left(w_{2}\right)$ follows from the following claim:

Claim. Under (4.2.6.1), the element $\left(\mathscr{G}_{1}^{-1}\left(d_{1}\right), \mathscr{G}_{2}^{-1}\left(d_{2}\right)\right)$ is sent to the element

$$
f_{\tilde{T}}^{-1}\left(\operatorname{span}_{\tilde{T}}\left\{v_{0} \otimes 1+w_{1} \otimes \epsilon_{1}+w_{2} \otimes \epsilon_{2}\right\}\right) .
$$

We now prove the claim. Let $\tilde{d}$ be such that the element $\left(\mathscr{G}_{1}^{-1}\left(d_{1}\right), \mathscr{G}_{2}^{-1}\left(d_{2}\right)\right)$ is sent under (4.2.6.1) to $f_{\tilde{T}}^{-1}(\tilde{d})$. Thus $\tilde{d}$ is an isotropic line in $(\Phi L)_{\tilde{T}}$. By the functoriality stated in Theorem 4.1.7 and the functorial definition of (4.2.6.1), we see that $\tilde{d}$ is characterized by the condition that $\tilde{d} \otimes_{\tilde{T}} T_{i}=d_{i}, i=1,2$, where the tensor product is with respect to the the structure map $\tilde{T} \rightarrow T_{i}$ expressing $\tilde{T}$ as the fiber product of $T_{1}, T_{2}$ (i.e. reduction modulo $\epsilon_{j}$ for $j \neq i$ ). Using this characterization of $\tilde{d}$, we see that $\tilde{d}$ is as predicted in the claim.

Corollary 4.2.7. The tangent space $\mathcal{T}_{x_{0}} \mathrm{RZ}_{k}$ is isomorphic to

$$
\left(\operatorname{Fil}^{1}(\Phi L)_{k}\right)^{\perp} / \operatorname{Fil}^{1}(\Phi L)_{k} .
$$

Under this isomorphism, the subspace $\mathcal{T}_{x_{0}} \mathcal{Z}(u)_{k}$ of $\mathcal{T}_{x_{0}} \mathrm{RZ}_{k}$ is identified with

$$
\left\{\bar{u}, \operatorname{Fil}^{1}(\Phi L)_{k}\right\}^{\perp} / \operatorname{Fil}^{1}(\Phi L)_{k} .
$$

Proof. This follows from Corollary 4.2.5, Lemma 4.2.6, and the bijectivity of $\mathscr{G}^{-1}$ asserted in Theorem 4.1.7.

Lemma 4.2.8. Let $\Lambda \subset V_{K}^{\Phi}$ be a vertex lattice. Let $L$ be a self-dual $W$-lattice in $V_{K}$ such that $\Lambda_{W}^{\vee} \subset L \subset \Lambda_{W}$. Let $A$ be the image of $\Lambda_{W}^{\vee}$ in $L_{k}$. Then the following statements hold. 
(1) $\operatorname{dim}_{k} \Lambda_{W} / L=\operatorname{dim}_{k} L / \Lambda_{W}^{\vee}$. Here both spaces are vector spaces over $k$ because $p \Lambda_{W} \subset \Lambda_{W}^{\vee} \subset L$ and $p L \subset p \Lambda_{W} \subset \Lambda_{W}^{\vee}$.

(2) $A \supset A^{\perp}$. Here $A^{\perp}$ is the orthogonal complement of $A$ in $L_{k}$.

Proof. (1) Consider the $W$-bilinear pairing

$$
\begin{gathered}
\Lambda_{W} \times \Lambda_{W} \rightarrow W \\
(x, y) \mapsto p\langle x, y\rangle,
\end{gathered}
$$

where $\langle$,$\rangle is the K$-bilinear form on $V_{K}^{\Phi} \otimes_{\mathbb{Q}_{p}} K=V_{K}$. We get an induced $k$-quadratic space structure on $\Lambda_{W} / \Lambda_{W}^{\vee}$. The image of $L$ in $\Lambda_{W} / \Lambda_{W}^{\vee}$ is equal to the orthogonal complement of itself, i.e. it is a Lagrangian subspace. Claim (1) follows.

(2) By definition $A^{\perp}$ is the image in $L_{k}$ of the $W$-submodule $p \Lambda_{W}^{\vee \vee}=p \Lambda_{W}$ of $L$. We have $p \Lambda_{W} \subset \Lambda_{W}^{\vee}$, so $A^{\perp}$ lies in the image of $\Lambda_{W}^{\vee}$ in $L_{k}$, which is $A$.

Proposition 4.2.9. Let $\Lambda \subset V_{K}^{\Phi}$ be a vertex lattice of type $t$ (so $t \geq 2$ is even). For all $x_{0} \in \mathrm{RZ}_{\Lambda}(k)$, we have

$$
\operatorname{dim}_{k} \mathcal{T}_{x_{0}} \mathrm{RZ}_{\Lambda, k}=t / 2-1
$$

Proof. Let $L$ be the special lattice associated to $x_{0}$ under (2.11.1.1), and let Fil ${ }^{1}(\Phi L)_{k}$ be as in Definition 4.1.3. Then $\Lambda_{W}^{\vee} \subset L \cap \Phi L$. Denote by $A$ the image of $\Lambda_{W}^{\vee}$ in $(\Phi L)_{k}$. Then $A$ is orthogonal to Fil ${ }^{1}(\Phi L)_{k}$ by Remark 4.1.8. By Corollary 4.2.7, we have an isomorphism of $k$-vector spaces

$$
\mathcal{T}_{x_{0}} \mathrm{RZ}_{\Lambda, k} \cong\left\{A, \operatorname{Fil}^{1}(\Phi L)_{k}\right\}^{\perp} / \operatorname{Fil}^{1}(\Phi L)_{k} .
$$

Since $A$ is orthogonal to $\mathrm{Fil}^{1}(\Phi L)_{k}$, we have $A \supset \mathrm{Fil}^{1}(\Phi L)_{k}$ by Lemma 4.2.8 applied to the self-dual $W$-lattice $\Phi L$. Therefore $\mathcal{T}_{x_{0}} \mathrm{RZ}_{\Lambda, k} \cong A^{\perp} / \mathrm{Fil}^{1}(\Phi L)_{k}$. Since the bilinear pairing on $(\Phi L)_{k}$ is non-degenerate, we have $\operatorname{dim}_{k} \mathcal{T}_{x_{0}} \mathrm{RZ}_{\Lambda, k}=\operatorname{dim}_{k}(\Phi L)_{k}-\operatorname{dim}_{k} A-1=\operatorname{dim}_{k}\left(\Phi L / \Lambda_{W}^{\vee}\right)-1$. By claim (1) in Lemma 4.2.8 (applied to $\Phi L)$, we have $\operatorname{dim}_{k}\left(\Phi L / \Lambda_{W}^{\vee}\right)=t / 2$.

Corollary 4.2.10. Let $\Lambda \subset V_{K}^{\Phi}$ be a vertex lattice. The formal scheme $\mathrm{RZ}_{\Lambda} \times_{\operatorname{Spf} W} \operatorname{Spec} k$ is regular.

Proof. Let $t$ be the type of $\Lambda$. Denote $X:=\mathrm{RZ}_{\Lambda}^{\mathrm{red}}$ and $Y:=\mathrm{RZ}_{\Lambda} \times_{\operatorname{Spf} W} \operatorname{Spec} k$. Then $X$ is a formal subscheme of $Y$ over $k$. Recall from $\$ 2.9$ that $X$ is a smooth $k$-scheme of dimension $t / 2-1$. It follows that for all $x_{0} \in Y(k)$, the complete local ring of $Y$ at $x_{0}$ is of dimension $\geq t / 2-1$. By Proposition 4.2.9, the tangent space of $Y$ at $x_{0}$ has $k$-dimension equal to $t / 2-1$. Hence $Y$ is regular at $x_{0}$.

Theorem 4.2.11. Let $\Lambda \subset V_{K}^{\Phi}$ be a vertex lattice. Then $\mathrm{RZ}_{\Lambda}=\mathrm{RZ}_{\Lambda}^{\mathrm{red}}$ and is of characteristic $p$.

Proof. $\mathrm{RZ}_{\Lambda}$ does not admit $W / p^{2}$-points (Proposition 4.2.1) and its special fiber is regular (Corollary 4.2.10). It follows from [RTZ13, Lemma 10.3] that $\mathrm{RZ}_{\Lambda}$ is equal to its special fiber. Being regular itself, $\mathrm{RZ}_{\Lambda}$ is reduced.

\section{The intersection Length Formula}

5.1. The arithmetic intersection as a fixed point scheme. Recall from 3.3 that we are interested in computing the intersection of $\mathrm{RZ}^{g}$ and $\delta\left(\mathrm{RZ}^{b}\right)$, for $g \in J_{b}\left(\mathbb{Q}_{p}\right)$.

Proposition 5.1.1. Assume $g \in J_{b}\left(\mathbb{Q}_{p}\right)$ is regular semisimple. Then $\delta\left(\mathrm{RZ}^{b}\right) \cap \mathrm{RZ}^{g}$ is contained in $\mathcal{Z}(\mathbf{v}(g))$, where $\mathbf{v}(g)=\left(x_{n}, g x_{n}, \cdots, g^{n-1} x_{n}\right)$. 
Proof. By Lemma 3.2.2, we have $\delta\left(\mathrm{RZ}^{b}\right) \subseteq \mathcal{Z}\left(x_{n}\right)$. Hence $\delta\left(\mathrm{RZ}^{b}\right) \cap \mathrm{RZ}^{g} \subseteq \mathcal{Z}\left(x_{n}\right) \cap \mathrm{RZ}^{g} \subseteq \mathcal{Z}\left(g x_{n}\right)$ by the definition of special cycles. Repeating this procedure we obtain

$$
\delta\left(\mathrm{RZ}^{\mathrm{b}}\right) \cap \mathrm{RZ}^{g} \subseteq \mathcal{Z}\left(x_{n}\right) \cap \mathcal{Z}\left(g x_{n}\right) \cap \cdots \cap \mathcal{Z}\left(g^{n-1} x_{n}\right)=\mathcal{Z}(\mathbf{v}(g)) .
$$

Corollary 5.1.2. Assume $g \in J_{b}\left(\mathbb{Q}_{p}\right)$ is regular semi-simple and minuscule. Then

$$
\delta\left(\mathrm{RZ}^{\mathrm{b}}\right) \cap \mathrm{RZ}^{g} \subset \mathrm{RZ}_{L(g)^{\vee}}=\mathrm{RZ}_{L(g)^{\vee}}^{\mathrm{red}} .
$$

In particular, $\delta\left(\mathrm{RZ}^{\mathrm{b}}\right) \cap \mathrm{RZ}^{g}$ is a scheme of characteristic $p$.

Proof. The first statement is an immediate consequence of Remark 2.14.4. Theorem 4.2.11 and Proposition 5.1.1. Now both $\delta\left(\mathrm{RZ}^{\mathrm{b}}\right)$ and $\mathrm{RZ}^{g}$ are closed formal subschemes of RZ, so $\delta\left(\mathrm{RZ}^{\mathrm{b}}\right) \cap \mathrm{RZ}^{g}$ is a closed formal subscheme of the scheme $\mathrm{RZ}_{L(g) \vee}=\mathrm{RZ}_{L(g)^{\vee}}^{\text {red }}$ of characteristic $p$. Hence $\delta\left(\mathrm{RZ}^{\mathrm{b}}\right) \cap \mathrm{RZ}^{g}$ is its self a scheme of characteristic $p$.

5.1.3. In the rest of this section we will fix $g \in J_{b}\left(\mathbb{Q}_{p}\right)$ regular semisimple and minuscule, and assume $\mathrm{RZ}^{g} \neq \varnothing$. Take $\Lambda:=L(g)^{\vee}$. Then $\Lambda$ is a vertex lattice stable under $g$, cf. Remark 3.6.1. We are interested in computing the intersection length of $\delta\left(\mathrm{RZ}^{\mathrm{b}}\right)$ and $\mathrm{RZ}^{g}$ around a $k$-point of intersection. Recall the isomorphism (2.9.1.1) between $p^{\mathbb{Z}} \backslash \mathrm{RZ} Z_{\Lambda}^{\text {red }}$ (which we now know is just $p^{\mathbb{Z}} \backslash \mathrm{RZ} Z_{\Lambda}$ ) and $S_{\Lambda}$. Recall from $\$ 2.8$ that $S_{\Lambda}$ is a projective smooth variety over $k$ of dimension $t_{\Lambda} / 2-1$. We write $d=t_{\Lambda} / 2$. Let $\Omega_{0}=\Lambda / \Lambda^{\vee}$ and $\Omega=\Omega_{0} \otimes_{\mathbb{F}_{p}} k=\Lambda_{W} / \Lambda_{W}^{\vee}$. Let $\langle$,$\rangle be the k$-bilinear form on $\Omega$ (cf. 2.7). Let $\mathbb{G}=\operatorname{SO}(\Omega), \mathbb{G}_{0}=\operatorname{SO}\left(\Omega_{0}\right)$. Let $\bar{g}$ be the induced action of $g$ on $\Omega$. Then $\bar{g} \in \mathbb{G}_{0}\left(\mathbb{F}_{p}\right) \subset \mathbb{G}(k)$.

There is a natural action of $\bar{g}$ on $S_{\Lambda}$ via its action on $\Omega$. On $R$-points $\bar{g}$ sends $\left(\mathcal{L}_{d-1}, \mathcal{L}_{d}\right)$ to $\left(\bar{g} \mathcal{L}_{d-1}, \bar{g} \mathcal{L}_{d}\right)$. The latter is indeed a point of $S_{\Lambda}$ because $\bar{g} \Phi=\Phi \bar{g}$ by the fact that $\bar{g} \in \mathbb{G}_{0}\left(\mathbb{F}_{p}\right)$. The following proposition allows us to reduce the study of intersection multiplicities to the study of the non-reduced structure of $S_{\Lambda}^{\bar{g}}$.

Proposition 5.1.4. $p^{\mathbb{Z}} \backslash\left(\delta\left(\mathrm{RZ}^{\mathrm{b}}\right) \cap \mathrm{RZ}^{g}\right) \cong S_{\Lambda}^{\bar{g}}$.

Proof. In view of Theorem 4.2.11, Corollary 5.1.2 and the observation that the isomorphism (2.9.1.1) induces an isomorphism $p^{\mathbb{Z}} \backslash\left(\mathrm{RZ}_{\Lambda}^{\text {red }}\right)^{g} \stackrel{\sim}{\longrightarrow} S_{\Lambda}^{\bar{g}}$, it suffices to show

$$
\left(p^{\mathbb{Z}} \backslash \mathrm{RZ}_{\Lambda}^{\mathrm{red}}\right) \cap\left(p^{\mathbb{Z}} \backslash \delta\left(\mathrm{RZ}^{\mathrm{b}}\right)\right)=\left(p^{\mathbb{Z}} \backslash \mathrm{RZ}_{\Lambda}^{\mathrm{red}}\right) .
$$

Since both $p^{\mathbb{Z}} \backslash \mathrm{RZ}_{\Lambda}^{\text {red }}$ and $p^{\mathbb{Z}} \backslash \delta\left(\mathrm{RZ}^{\mathrm{b}}\right)$ are closed formal subschemes of $p^{\mathbb{Z}} \backslash \mathrm{RZ}$ and since $p^{\mathbb{Z}} \backslash \mathrm{RZ} \mathbf{L}_{\Lambda}^{\text {red }}$ is a reduced scheme, it suffices to check that

$$
p^{\mathbb{Z}} \backslash \mathrm{RZ}_{\Lambda}^{\text {red }}(k) \subset p^{\mathbb{Z}} \backslash \delta\left(\mathrm{RZ}^{b}\right)(k) .
$$

Now the left hand side consists of special lattices $L$ containing $\Lambda^{\vee}$, and the right hand side consists of special lattices $L$ containing $x_{n}$ (cf. (2.12.0.1) and Lemma 3.4.2). We finish the proof by noting that by definition $x_{n} \in \Lambda^{\vee}=L(g)$.

Proposition 5.1 .4 reduces the intersection problem to the study of $S_{\Lambda}^{\bar{g}}$.

5.2. Study of $S_{\Lambda}^{\bar{g}}$. We continue to use the notation in \$5.1. We adopt the following notation from [HP14, $\S 3.2]$.

Definition 5.2.1. Let $\operatorname{OGr}(d-1)$ (resp. $\operatorname{OGr}(d)$ ) be the moduli space of totally isotropic subspaces of $\Omega$ of dimension $d-1$ (resp. $d$ ). For a finite dimensional vector space $W$ over $k$ and an integer $l$ with $0 \leq l \leq \operatorname{dim} W$, we write $\operatorname{Gr}(W, l)$ for the Grassmannian classifying $l$-dimensional subspaces of $W$. Thus for $j \in\{d-1, d\}$ and any $k$-algebra $R$, we have

$\operatorname{OGr}(j)(R)=\left\{R\right.$-module local direct summands of $\Omega \otimes_{k} R$ of local rank $j$ which are totally isotropic $\}$. 
Also

$$
\operatorname{Gr}(W, l)(R)=\left\{R \text {-module local direct summands of } W \otimes_{k} R \text { of local rank } k\right\} .
$$

Definition 5.2.2. If $A$ is a finite dimensional $k$-vector space, we write $\mathbb{A}^{A}$ for the affine space over $k$ defined by $A$. Thus for a $k$-algebra $R$ we have $\mathbb{A}^{A}(R)=A \otimes_{k} R$.

Definition 5.2.3. Let $\mathcal{L}_{d}, \mathcal{M}_{d}$ be Lagrangian subspaces of $\Omega$ such that $\Omega=\mathcal{L}_{d} \oplus \mathcal{M}_{d}$. we write $\operatorname{Hom}_{\text {anti }}\left(\mathcal{L}_{d}, \mathcal{M}_{d}\right)$ for the space of anti-symmetric $k$-linear maps $\mathcal{L}_{d} \rightarrow \mathcal{M}_{d}$. Here we say $\phi: \mathcal{L}_{d} \rightarrow \mathcal{M}_{d}$ is anti-symmetric if the bilinear form $\mathcal{L}_{d} \times \mathcal{L}_{d} \rightarrow k,(x, y) \mapsto\langle x, \phi y\rangle$ is anti-symmetric.

5.2.4. Recall that in general, if $A$ is a finite dimensional vector space over $k$ and $B$ is a subspace, then we can construct a Zariski open of the $\operatorname{Grassmannian} \operatorname{Gr}(A, \operatorname{dim} B)$ as follows. Choose a subspace $C$ of $A$ such that $A=B \oplus C$. Then there is an open embedding $\iota_{B, C}: \mathbb{A}^{\operatorname{Hom}_{k}(B, C)} \rightarrow \operatorname{Gr}(A, \operatorname{dim} B)$ which we now describe. For any $k$-algebra $R$ and any $R$-point $\phi$ of $\mathbb{A}^{\operatorname{Hom}_{k}(B, C)}$, we view $\phi$ as an element of $\operatorname{Hom}_{k}(B, C) \otimes R=\operatorname{Hom}_{R}(B \otimes R, C \otimes R)$. Then $\iota_{B, C}$ maps $\phi$ to the $R$-point of $\operatorname{Gr}(A, \operatorname{dim} B)$ corresponding the following $R$-submodule of $A$ :

$$
\{x+\phi(x) \mid x \in B \otimes R\} .
$$

For details see for instance [Har95, Lecture 6]. In the following we will think of $\mathbb{A}^{\operatorname{Hom}_{k}(B, C)}$ as a Zariski open of $\operatorname{Gr}(A, \operatorname{dim} B)$, omitting $\iota_{B, C}$ from the notation.

Lemma 5.2.5. Let $\mathcal{L}_{d}, \mathcal{M}_{d}$ be complementary Lagrangian subspaces of $\Omega$ over $k$. Then

$$
\operatorname{OGr}(d) \times_{\operatorname{Gr}(\Omega, d)} \mathbb{A}^{\operatorname{Hom}\left(\mathcal{L}_{d}, \mathcal{M}_{d}\right)}=\mathbb{A}^{\operatorname{Hom}_{\text {anti }}\left(\mathcal{L}_{d}, \mathcal{M}_{d}\right)} .
$$

In particular, the k-point $\mathcal{L}_{d}$ in $\operatorname{OGr}(d)$ has an open neighborhood of the form $\mathbb{A}^{\operatorname{Hom}_{\text {anti }}\left(\mathcal{L}_{d}, \mathcal{M}_{d}\right)}$.

Proof. Let $R$ be a $k$-algebra and $\phi$ an $R$-point of $\mathbb{A}^{\operatorname{Hom}\left(\mathcal{L}_{d}, \mathcal{M}_{d}\right)}$. Then the submodule (5.2.4.1) (for $B=\mathcal{L}_{d}$ ) is Lagrangian if and only if for all $x \in B \otimes R$,

$$
\langle x+\phi(x), x+\phi(x)\rangle=0
$$

But we have $\langle x, x\rangle=\langle\phi(x), \phi(x)\rangle=0$ since $\mathcal{L}_{d} \otimes R$ and $\mathcal{M}_{d} \otimes R$ are both Lagrangian. Hence (5.2.4.1) is Lagrangian if and only if $\langle x, \phi(x)\rangle=0$ for all $x \in \mathcal{M}_{d} \otimes R$.

5.2.6. It follows from the assumptions we made on $\bar{g} \in \mathbb{G}(k)$ in 5.1 .3 that its characteristic polynomial on $\Omega$ is equal to its minimal polynomial on $\Omega$ (cf. Remark 3.6.1). In general this property is equivalent to the property that in the Jordan normal form all the Jordan blocks have distinct eigenvalues. From now on we let $x_{0}=\left(\mathcal{L}_{d-1}, \mathcal{L}_{d}\right) \in S_{\Lambda}(k)$ be an element fixed by $\bar{g}$. Then $\Phi \mathcal{L}_{d} \subset \Omega$ is also stable under $\bar{g}$. If we identify $\Omega / \Phi \mathcal{L}_{d}$ with $\left(\Phi \mathcal{L}_{d}\right)^{*}$ (the $k$-vector space dual) using the bilinear form on $\Omega$, the action of $\bar{g}$ on $\Omega / \Phi \mathcal{L}_{d}$ is equal to the inverse transpose of $\left.\bar{g}\right|_{\Phi \mathcal{L}_{d}}$. It follows that the minimal polynomial (resp. characteristic polynomial) of $\bar{g}$ on $\Omega$ is equal to the minimal polynomial (resp. characteristic polynomial) of $\left.\bar{g}\right|_{\Phi \mathcal{L}_{d}}$ times its reciprocal. Hence $\left.\bar{g}\right|_{\Phi \mathcal{L}_{d}}$ has equal minimal and characteristic polynomial, too.

Definition 5.2.7. Let $\lambda$ be the (nonzero) eigenvalue of $\bar{g}$ on the one-dimensional $\Phi \mathcal{L}_{d} / \mathcal{L}_{d-1}$. Let $c$ be the size of the unique Jordan block of eigenvalue $\lambda$ of $\left.\bar{g}\right|_{\Phi \mathcal{L}_{d}}$. 
5.2.8. Let $x_{0}=\left(\mathcal{L}_{d-1}, \mathcal{L}_{d}\right) \in S_{\Lambda}(k)^{\bar{g}}$ as in 5.2.6. Define

$$
Y:=\operatorname{Gr}\left(\Phi \mathcal{L}_{d}, d-1\right) \times_{k} \operatorname{OGr}(d) .
$$

Let $\mathcal{I} \subset Y$ be the sub-functor defined by the incidence relation, i.e. for a $k$-algebra $R$

$$
\mathcal{I}(R)=\left\{\left(\mathcal{L}_{d-1}^{\prime}, \mathcal{L}_{d}^{\prime}\right) \in \operatorname{Gr}\left(\Phi \mathcal{L}_{d}, d-1\right)(R) \times \operatorname{OGr}(d)(R) \mid \mathcal{L}_{d-1}^{\prime} \subset \mathcal{L}_{d}^{\prime}\right\} .
$$

The pair $\left(\mathcal{L}_{d-1}, \mathcal{L}_{d}\right)$ defines a $k$-point in $\mathcal{I}$, which we again denote by $x_{0}$. It is well known that the incidence sub-functor of $\operatorname{Gr}\left(\Phi \mathcal{L}_{d}, d-1\right) \times \operatorname{Gr}(\Omega, d)$ is represented by a closed subscheme, and it follows that $\mathcal{I}$ is a closed subscheme of $Y$.

Since $x_{0}=\left(\mathcal{L}_{d-1}, \mathcal{L}_{d}\right) \in S_{\Lambda}(k)$ is fixed by $\bar{g}$, we have a natural action of $\bar{g}$ on $Y$, stabilizing $\mathcal{I}$ and fixing $x_{0} \in \mathcal{I}$. Let

$$
\tilde{\mathcal{R}}:=\mathcal{O}_{\mathcal{I}, x_{0}}, \quad \mathcal{R}:=\mathcal{O}_{\mathcal{I}^{\bar{g}}, x_{0}}, \quad \tilde{\mathcal{S}}:=\mathcal{O}_{S_{\Lambda}, x_{0}}, \quad \mathcal{S}:=\mathcal{O}_{S_{\Lambda}^{\bar{g}}, x_{0}}
$$

be the local rings at $x_{0}$ of $\mathcal{I}, \mathcal{I}^{\bar{g}}, S_{\Lambda}, S_{\Lambda}^{\bar{g}}$ respectively. Let

$$
\tilde{\mathcal{R}}_{p}:=\tilde{\mathcal{R}} / \mathfrak{m}_{\tilde{\mathcal{R}}}^{p}, \quad \mathcal{R}_{p}:=\mathcal{R} / \mathfrak{m}_{\mathcal{R}}^{p}, \quad \tilde{\mathcal{S}}_{p}:=\tilde{\mathcal{S}} / \mathfrak{m}_{\tilde{\mathcal{S}}}^{p}, \quad \mathcal{S}_{p}:=\mathcal{S} / \mathfrak{m}_{\mathcal{S}}^{p}
$$

be the above four local rings modulo the $p$-th powers of their respective maximal ideals.

The following lemma expresses the observation that $\mathcal{I}^{\bar{g}}$ may serve as a model for $S_{\Lambda}^{\bar{g}}$ locally around $x_{0}$.

Lemma 5.2.9. (1) There is a k-algebra isomorphism $\tilde{\mathcal{R}}_{p} \cong \tilde{\mathcal{S}}_{p}$, equivariant for the $\bar{g}$-action on both sides.

(2) There is a k-algebra isomorphism $\mathcal{R}_{p} \cong \mathcal{S}_{p}$.

Proof. We first show (1). Let $\left(\mathcal{L}_{d-1}^{\prime}, \mathcal{L}_{d}^{\prime}\right)$ be the tautological pair over $\tilde{\mathcal{S}}_{p}$ for the moduli problem $S_{\Lambda}$, and let $\left(\mathcal{L}_{d-1}^{\prime \prime}, \mathcal{L}_{d}^{\prime \prime}\right)$ be the tautological pair over $\tilde{\mathcal{R}}_{p}$ for the moduli problem $\mathcal{I}$. Note that

$$
\Phi \mathcal{L}_{d}^{\prime}=\left(\Phi \mathcal{L}_{d}\right) \otimes \tilde{\mathcal{S}}_{p}
$$

as submodules of $\Omega \otimes_{k} \tilde{\mathcal{S}}_{p}$ because $\Phi: \tilde{\mathcal{S}}_{p} \rightarrow \tilde{\mathcal{S}}_{p}$ factors through the reduction map $\tilde{\mathcal{S}}_{p} \rightarrow k$. It follows that $\left(\mathcal{L}_{d-1}^{\prime}, \mathcal{L}_{d}^{\prime}\right)$ defines a point in $\mathcal{I}\left(\tilde{\mathcal{S}}_{p}\right)$ lifting $x_{0} \in \mathcal{I}(k)$. Similarly,

$$
\Phi \mathcal{L}_{d}^{\prime \prime}=\left(\Phi \mathcal{L}_{d}\right) \otimes \tilde{\mathcal{R}}_{p}
$$

as submodules of $\Omega \otimes_{k} \tilde{\mathcal{R}}_{p}$, and hence $\left(\mathcal{L}_{d-1}^{\prime \prime}, \mathcal{L}_{d}^{\prime \prime}\right)$ defines a point in $S_{\Lambda}\left(\tilde{\mathcal{R}}_{p}\right)$ lifting $x_{0} \in \mathcal{S}_{\Lambda}(k)$. The point in $\mathcal{I}\left(\tilde{\mathcal{S}}_{p}\right)$ and the point in $S_{\Lambda}\left(\tilde{\mathcal{R}}_{p}\right)$ constructed above give rise to inverse $k$-algebra isomorphisms between $\tilde{\mathcal{R}}_{p}$ and $\tilde{\mathcal{S}}_{p}$, which are obviously $\bar{g}$-equivariant.

(2) follows from $(1)$, since $\mathcal{R}_{p}$ (resp. $\mathcal{S}_{p}$ ) is the quotient ring of $\tilde{\mathcal{R}}_{p}$ (resp. $\tilde{\mathcal{S}}_{p}$ ) modulo the ideal generated by elements of the form $r-\bar{g} \cdot r$ with $r \in \tilde{\mathcal{R}}_{p}$ (resp. $r \in \tilde{\mathcal{S}}_{p}$ ).

5.3. Study of $\mathcal{I}^{\bar{g}}$. Next we study $\mathcal{I}^{\bar{g}}$ by choosing certain explicit coordinates on $\mathcal{I}$. Choose a $k$-basis $v_{1}, \cdots, v_{d}, w_{1}, \cdots, w_{d}$ of $\Omega$, such that

- $\mathcal{L}_{d-1}$ is spanned by $v_{1}, \cdots, v_{d-1}$.

- $\mathcal{L}_{d}$ is spanned by $v_{1}, \cdots, v_{d}$.

- $\Phi \mathcal{L}_{d}$ is spanned by $v_{1}, \cdots, v_{d-1}, w_{d}$.

- $\left\langle v_{i}, v_{j}\right\rangle=\left\langle w_{i}, w_{j}\right\rangle=0,\left\langle v_{i}, w_{j}\right\rangle=\delta_{i j}$.

We will denote

Also denote

$$
\hat{v}_{i}:=\left\{\begin{array}{l}
v_{i}, 1 \leq i \leq d-1 \\
w_{d}, i=d
\end{array}\right.
$$

$$
\mathcal{M}_{d}:=\operatorname{span}_{k}\left(w_{1}, \cdots, w_{d}\right) \text {. }
$$


For $1 \leq i \leq d-1$, define an element $\phi_{i} \in \operatorname{Hom}\left(\mathcal{L}_{d-1}, \operatorname{span}_{k}\left(w_{d}\right)\right)$ by

$$
\phi_{i}\left(v_{j}\right)=\delta_{i j} w_{d}
$$

Then $\phi_{1}, \cdots, \phi_{d-1}$ is a basis of $\operatorname{Hom}\left(\mathcal{L}_{d-1}, \operatorname{span}_{k}\left(w_{d}\right)\right)$.

By $\$ 5.2 .4$ and Lemma 5.2.5 there is a Zariski open neighborhood of $x_{0}$ in $Y$, of the form

$$
\mathcal{U}:=\mathbb{A}^{\operatorname{Hom}\left(\mathcal{L}_{d-1}, \operatorname{span}_{k}\left(w_{d}\right)\right)} \times \mathbb{A}^{\operatorname{Hom}_{\mathrm{anti}}\left(\mathcal{L}_{d}, \mathcal{M}_{d}\right)} .
$$

Lemma 5.3.1. (1) Let $R$ be a $k$-algebra. Let $y \in \mathcal{U}(R)$, corresponding to

$$
(\phi, \psi) \in \operatorname{Hom}\left(\mathcal{L}_{d-1}, \operatorname{span}_{k}\left(w_{d}\right)\right) \otimes R \oplus \operatorname{Hom}_{\text {anti }}\left(\mathcal{L}_{d}, \mathcal{M}_{d}\right) \otimes R .
$$

We view $\phi \in \operatorname{Hom}_{R}\left(\mathcal{L}_{d-1} \otimes R, \operatorname{span}_{R}\left(w_{d}\right)\right)$ and $\psi \in \operatorname{Hom}_{R}\left(\mathcal{L}_{d} \otimes R, \mathcal{M}_{d} \otimes R\right)$. Then $y$ is in $\mathcal{I}$ if and only if $\left.\psi\right|_{\mathcal{L}_{d-1} \otimes R}=\phi$.

(2) The projection to the first factor $\mathcal{U} \rightarrow \mathbb{A}^{\operatorname{Hom}\left(\mathcal{L}_{d-1}, \operatorname{span}_{k}\left(w_{d}\right)\right)}$ restricts to an isomorphism

$$
\mathcal{U} \cap \mathcal{I} \stackrel{\sim}{\longrightarrow} \mathbb{A}^{\operatorname{Hom}\left(\mathcal{L}_{d-1}, \operatorname{span}_{k}\left(w_{d}\right)\right)} .
$$

Proof. (1) We know that $y$ is in $\mathcal{I}$ if and only if for all $v \in \mathcal{L}_{d-1} \otimes R$, there exists $v^{\prime} \in \mathcal{L}_{d} \otimes R$, such that

$$
v+\phi(v)=v^{\prime}+\psi\left(v^{\prime}\right)
$$

as elements of $\Omega \otimes R$. Decompose $v^{\prime}=v_{1}^{\prime}+v_{2}^{\prime}$ with $v_{1}^{\prime} \in \mathcal{L}_{d-1} \otimes R$ and $v_{2}^{\prime} \in \operatorname{span}_{R}\left(v_{d}\right)$. Then the above equation reads

$$
v-v_{1}^{\prime}=v_{2}^{\prime}+\left(\psi\left(v^{\prime}\right)-\phi(v)\right) .
$$

Since $v-v_{1}^{\prime} \in \mathcal{L}_{d-1} \otimes R, v_{2}^{\prime} \in \operatorname{span}_{R}\left(v_{d}\right), \psi\left(v^{\prime}\right)-\phi(v) \in \mathcal{M}_{d} \otimes R$, the above equation holds if and only if $v=v_{1}^{\prime}, v_{2}^{\prime}=0, \phi(v)=\psi(v)$. Hence $y \in \mathcal{I}$ if and only if for all $v \in \mathcal{L}_{d-1} \otimes R$ we have $\psi(v)=\phi(v)$. This proves (1).

(2) By (1), we know that $\mathcal{U} \cap \mathcal{I}$ is the affine subspace of $\mathcal{U}$ associated to the linear subspace of

$$
\operatorname{Hom}\left(\mathcal{L}_{d-1}, \operatorname{span}_{k}\left(w_{d}\right)\right) \times \operatorname{Hom}_{\text {anti }}\left(\mathcal{L}_{d}, \mathcal{M}_{d}\right)
$$

consisting of pairs $(\phi, \psi)$ such that $\left.\psi\right|_{\mathcal{L}_{d-1}}=\phi$. Call this subspace $A$. We only need to show that projection to the first factor induces an isomorphism $A \stackrel{\sim}{\longrightarrow} \operatorname{Hom}\left(\mathcal{L}_{d-1}, \operatorname{span}_{k}\left(w_{d}\right)\right)$.

Note that if $\psi \in \operatorname{Hom}_{\text {anti }}\left(\mathcal{L}_{d}, \mathcal{M}_{d}\right)$, then $\psi$ is determined by $\left.\psi\right|_{\mathcal{L}_{d-1}}$. This is because for each $1 \leq i \leq d$, we have

$$
\left\langle\psi v_{d}, v_{i}\right\rangle=\left\{\begin{array}{l}
-\left\langle v_{d}, \psi v_{i}\right\rangle, i \leq d-1 \\
0, i=d
\end{array}\right.
$$

which means that $\psi\left(v_{d}\right)$ is determined by $\left.\psi\right|_{\mathcal{L}_{d-1}}$. Conversely, given $\phi \in \operatorname{Hom}\left(\mathcal{L}_{d-1}, \operatorname{span}_{k}\left(w_{d}\right)\right)$ we can construct $\psi \in \operatorname{Hom}_{\text {anti }}\left(\mathcal{L}_{d}, \mathcal{M}_{d}\right)$ such that $\left.\psi\right|_{\mathcal{L}_{d-1}}=\phi$ as follows. For $1 \leq j \leq d-1$, define $\psi\left(v_{j}\right)$ to be $\phi\left(v_{j}\right)$. Define $\psi\left(v_{d}\right)$ to be the unique element of $\mathcal{M}_{d}$ satisfying (5.3.1.1). In this way we have defined a linear map $\psi: \mathcal{L}_{d} \rightarrow \mathcal{M}_{d}$ such that $\left.\psi\right|_{\mathcal{L}_{d-1}}=\phi$. We now check that $\psi$ is anti-symmetric. We need to check that for all $1 \leq i \leq j \leq d$, we have $\left\langle\psi v_{j}, v_{i}\right\rangle=-\left\langle\psi v_{i}, v_{j}\right\rangle$. If $j=d$, this is true by (5.3.1.1). Suppose $j<d$. Then $\left\langle\psi v_{j}, v_{i}\right\rangle=\left\langle\psi v_{i}, v_{j}\right\rangle=0$ because $\psi v_{j}, \psi v_{i} \in \operatorname{span}_{k}\left(w_{d}\right)$ and $\left\langle w_{d}, \mathcal{L}_{d-1}\right\rangle=0$. Thus $\psi$ is indeed antisymmetric. It follows that $A \stackrel{\sim}{\longrightarrow} \operatorname{Hom}\left(\mathcal{L}_{d-1}, \operatorname{span}_{k}\left(w_{d}\right)\right)$.

From now on we assume $x_{0}=\left(\mathcal{L}_{d-1}, \mathcal{L}_{d}\right) \in S_{\Lambda}^{\bar{g}}(k)$. 
Definition 5.3.2. Write the matrix over $k$ of $\bar{g}$ acting on $\Phi \mathcal{L}_{d}$ under the basis $\hat{v}_{1}, \cdots, \hat{v}_{d}$ (cf. $\left.\$ 5.3\right)$ as

$$
\left(\begin{array}{ll}
H_{1} & H_{2} \\
H_{3} & H_{4}
\end{array}\right)
$$

where $H_{1}$ is of size $(d-1) \times(d-1), H_{2}$ is of size $(d-1) \times 1, H_{3}$ is of size $1 \times(d-1)$, and $H_{4} \in k$.

Remark 5.3.3. Since $\bar{g}$ stabilizes $\mathcal{L}_{d-1}$, we have $H_{3}=0$

Proposition 5.3.4. Let $R$ be a $k$-algebra and let $y=(\phi, \psi) \in \mathcal{U}(R)$. Represent $\phi$ as an R-linear combination $\phi=\sum_{i=1}^{d-1} r_{i} \phi_{i}$ of the $\phi_{i}$ 's (cf. (5.3.0.1)), where $r_{i} \in R$. Write $\vec{r}$ for the row vector $\left(r_{1}, \cdots, r_{d-1}\right)$.

(1) View $\phi$ as an element of $\operatorname{Gr}\left(\Phi \mathcal{L}_{d}, d-1\right)(R)$. It is fixed by $\left.\bar{g}\right|_{\Phi \mathcal{L}_{d}}$ if and only if

$$
\vec{r}\left(H_{1}+H_{2} \vec{r}\right)=H_{4} \vec{r} .
$$

(2) Assume that $y \in \mathcal{I}(R)$ and that $\phi \in \operatorname{Gr}\left(\Phi \mathcal{L}_{d}, d-1\right)$ is fixed by $\left.\bar{g}\right|_{\Phi \mathcal{L}_{d}}$. Then $\psi$, viewed as an element of $\operatorname{OGr}(d)(R)$, is fixed by $\bar{g}$. In other words, $y$ is fixed by $\bar{g}$ in this case.

Proof. (1) First we identify $\left(\Phi \mathcal{L}_{d}\right) \otimes R$ with $R^{d-1}$ using the basis $\hat{v}_{1}, \cdots, \hat{v}_{d}$. As a point of $\operatorname{Gr}\left(\Phi \mathcal{L}_{d}, d-1\right)$, $\phi$ corresponds to the following submodule of $\left(\Phi \mathcal{L}_{d}\right) \otimes R$ : the image, i.e. column space, of the $R$-matrix

$$
\left(\begin{array}{cc}
I_{d-1} & 0 \\
\vec{r} & 0
\end{array}\right)
$$

Hence $\phi \in \operatorname{Gr}\left(\Phi \mathcal{L}_{d}, d-1\right)$ is fixed by $\left.\bar{g}\right|_{\Phi \mathcal{L}_{d}}$ if and only if the following two $R$-matrices have the same column space:

$$
A_{1}:=\left(\begin{array}{cc}
I_{d-1} & 0 \\
\vec{r} & 0
\end{array}\right) \text { and } A_{2}:=\left(\begin{array}{cc}
H_{1} & H_{2} \\
H_{3} & H_{4}
\end{array}\right)\left(\begin{array}{cc}
I_{d-1} & 0 \\
\vec{r} & 0
\end{array}\right) .
$$

Note that since $\left(\begin{array}{ll}H_{1} & H_{2} \\ H_{3} & H_{4}\end{array}\right)$ is invertible, $A_{1}$ and $A_{2}$ have the same column space if and only if the column space of $A_{2}$ is contained in that of $A_{1}$. Since $H_{3}=0$ (cf. Remark 5.3.3), we have

$$
A_{2}=\left(\begin{array}{cc}
H_{1}+H_{2} \vec{r} & 0 \\
H_{4} \vec{r} & 0
\end{array}\right)
$$

But we easily see that the column space of $\left(\begin{array}{cc}H_{1}+H_{2} \vec{r} & 0 \\ H_{4} \vec{r} & 0\end{array}\right)$ is contained in that of $\left(\begin{array}{cc}I_{d-1} & 0 \\ \vec{r} & 0\end{array}\right)$ if and only if (5.3.4.1) holds.

(2) Let $\operatorname{OGr}(d-1, d)$ be the incidence subscheme of $\operatorname{OGr}(d-1) \times \operatorname{OGr}(d)$. Consider the natural morphism $f: \mathcal{I} \rightarrow \operatorname{OGr}(d-1, d),\left(\mathcal{L}_{d-1}^{\prime}, \mathcal{L}_{d}^{\prime}\right) \mapsto\left(\mathcal{L}_{d-1}^{\prime}, \mathcal{L}_{d}^{\prime}\right)$. Note that $\mathcal{U} \cap \mathcal{I}$ is connected because it is a linear subspaces of the affine spaces $\mathcal{U}$ (cf. Lemma 5.3.1). Thus $(\bar{g} \cdot \mathcal{U}) \cap \mathcal{I}=\bar{g}(\mathcal{U} \cap \mathcal{I})$ is also connected. Since $\mathcal{U} \cap \mathcal{I}$ and $(\bar{g} \cdot \mathcal{U}) \cap \mathcal{I}$ share a common $k$-point, namely $x_{0}$, we see that that $f(\mathcal{U} \cap \mathcal{I})$ and $f((\bar{g} \cdot \mathcal{U}) \cap \mathcal{I})$ are in one connected component of $\operatorname{OGr}(d-1, d)$. We have $y \in \mathcal{U} \cap \mathcal{I}$ and $\bar{g} y \in(\bar{g} \cdot \mathcal{U}) \cap \mathcal{I}$. In particular $f(y)$ and $f(\bar{g} y)$ are $R$-points of the aforementioned connected component of $\operatorname{OGr}(d-1, d)$. Recall from [HP14, §3.2] that $\operatorname{OGr}(d-1, d)$ has two connected components, and each is isomorphic to $\operatorname{OGr}(d-1)$ via the projection to the first factor. Our assumptions imply that $f(y), f(\bar{g} y)$ have the same image in $\operatorname{OGr}(d-1)$. It follows that $f(y)=f(\bar{g} y)$. But by definition $f$ is injective on $R$-points, so $y=\bar{g} y$. 
Proposition 5.3.5. Assume $x_{0} \in S_{\Lambda}^{\bar{g}}(k)$. Then the local ring $\mathcal{R}=\mathcal{O}_{\mathcal{I}^{\bar{g}}, x_{0}}$ of $\mathcal{I}^{\bar{g}}$ at $x_{0}$, is isomorphic to the local ring at the origin of the subscheme of $\mathbb{A}_{k}^{d-1}$ defined by the equations 5.3.4.1), where $\mathbb{A}_{k}^{d-1}$ has coordinates $r_{1}, \cdots, r_{d-1}$. Moreover, explicitly we have

$$
\mathcal{R} \cong k[X] / X^{c} .
$$

Proof. The first claim follows from Lemma 5.3.1 and Proposition 5.3.4. To compute $\mathcal{R}$ explicitly, we may and shall assume that the bases chosen in 5.2.8 are such that the matrix $H_{1}$ is already in its (upper-triangular) Jordan normal form. Recall from Definition 5.2.7 that all the Jordan blocks have distinct eigenvalues. Let $J_{d_{1}}\left(\lambda_{1}\right), \cdots, J_{d_{s-1}}\left(\lambda_{s-1}\right)$ be the Jordan blocks that have eigenvalues different from $\lambda$. Let $\lambda_{s}=\lambda$ and let $J_{d_{s}}\left(\lambda_{s}\right)$ be the Jordan block of eigenvalue $\lambda_{s}$ that appears in $H_{1}$, where we allow $d_{s}=0$. Then $d_{s}=c-1$. Moreover, we assume that $J_{d_{1}}\left(\lambda_{1}\right), \cdots, J_{d_{s}}\left(\lambda_{s}\right)$ appear in the indicated order. Note that $H_{4}=\lambda$. Write $H_{1}=\left(h_{i j}\right)_{1 \leq i, j \leq d-1}$. The equations (5.3.4.1) become

$$
\left\{\begin{array}{l}
r_{i-1} h_{i-1, i}+\left(h_{i, i}-\lambda+\vec{r} H_{2}\right) r_{i}=0,2 \leq i \leq d-1 \\
\left(h_{1,1}-\lambda+\vec{r} H_{2}\right) r_{1}=0
\end{array}\right.
$$

Note that when $h_{i, i}$ is not in the Jordan block $J_{d_{s}}\left(\lambda_{s}\right)$, we have $h_{i, i}-\lambda \in k^{\times}$, so the element $h_{i, i}-\lambda+\vec{r} H_{2}$ is a unit in the local ring $\mathcal{O}_{\mathbb{A}^{d-1}, 0}$. Hence for $i \leq d_{1}+d_{2}+\cdots+d_{s-1}=d-c$, each $r_{i}$ is solved to be a multiple of $r_{i-1}$ and this multiple eventually becomes zero when this procedure is iterated. In other words, the ideal in $\mathcal{O}_{\mathbb{A}^{d-1}, 0}$ defining $\mathcal{R}$ is generated by

$$
r_{1}, r_{2}, \cdots, r_{d-c}, \quad\left(\vec{r} H_{2}\right) r_{d-c+1}, \quad\left(\vec{r} H_{2}\right) r_{i}+r_{i-1}(d-c+1<i \leq d-1) .
$$

When $c=1$, we have $\mathcal{R} \cong k$ as expected. Assume now $c \geq 2$. Let $h_{1}, \cdots, h_{c-1}$ be the last $c-1$ entries of the $(d-1) \times 1$-matrix $H_{2}$. Make the change of variables

$$
\left\{\begin{array}{l}
X_{i}=r_{d-c+i}, 1 \leq i \leq c-1 \\
A=\vec{r} H_{2}
\end{array}\right.
$$

Then we have

$$
\mathcal{R} \cong\left(\frac{k\left[X_{1}, \cdots, X_{c-1}, A\right]}{\left(A-\sum_{i=1}^{c-1} h_{i} X_{i}, A X_{1}, X_{1}+A X_{2}, X_{2}+A X_{3}, \cdots, X_{c-2}+A X_{c-1}\right)}\right)_{\left(X_{1}, \cdots, X_{c-1}\right)}
$$

By eliminating the variables $X_{1}, \ldots, X_{c-2}$, we obtain that

$$
\mathcal{R} \cong\left(\frac{k\left[X_{c-1}, A\right]}{\left(X_{c-1} A^{c-1}, A-X_{c-1} \sum_{i=0}^{c-2} h_{c-1-i}(-A)^{i}\right)}\right)_{\left(X_{c-1}, A\right)} .
$$

Note that if $h_{c-1}=0$, then the last two rows of the matrix

$$
\lambda I_{d}-\left(\begin{array}{cc}
H_{1} & H_{2} \\
0 & H_{4}
\end{array}\right)
$$

are both zero. This contradicts with the fact that the matrix $\left(\begin{array}{cc}H_{1} & H_{2} \\ 0 & H_{4}\end{array}\right)$, which represents $\bar{g}$ on $\Phi \mathcal{L}_{d}$, has in its Jordan normal form a unique Jordan block of eigenvalue $\lambda$ (cf. \$5.2.6). Hence $h_{c-1} \neq 0$, and $\sum_{i=0}^{c-2} h_{c-1-i}(-A)^{i}$ is a unit in $k\left[X_{c-1}, A\right]_{\left(X_{c-1}, A\right)}$. It follows that

$$
\mathcal{R} \cong\left(\frac{k[X]}{\left(X^{c}\right)}\right)_{(X)}=k[X] / X^{c}
$$


as desired.

5.4. The intersection length formula. We are now ready to determine the structure of the complete local ring of $S_{\Lambda}^{\bar{g}}$ at a $k$-point of it, when $p$ is large enough. It is a consequence of Lemma 5.2.9. Proposition 5.3.5, and some commutative algebra.

Theorem 5.4.1. Let $x_{0} \in S_{\Lambda}^{\bar{g}}(k)$. Let $\lambda$ and $c$ be as in Definition 5.2.7. Assume $p>c$. Then the complete local ring of $S_{\Lambda}^{\bar{g}}$ at $x_{0}$ is isomorphic to $k[X] / X^{c}$.

Proof. Since $S_{\Lambda}$ is smooth of dimension $d-1$ (cf. 82.8 ), the complete local ring of $S_{\Lambda}^{\bar{g}}$ at $x_{0}$ is of the form

$$
\hat{\mathcal{S}}=k\left[\left[X_{1}, \cdots, X_{d-1}\right]\right] / I
$$

for a proper ideal $I$ of $k\left[\left[X_{1}, \cdots, X_{d-1}\right]\right] .5$ Let $\mathfrak{m}$ be the maximal ideal of $k\left[\left[X_{1}, \cdots, X_{d-1}\right]\right]$ and let $\overline{\mathfrak{m}}$ be the maximal ideal of $\hat{\mathcal{S}}$. By Lemma 5.2.9 and Proposition 5.3.5, there is an isomorphism

$$
\beta: \hat{\mathcal{S}} / \overline{\mathfrak{m}}^{p} \stackrel{\sim}{\longrightarrow} k[X] / X^{c} .
$$

We first notice that if $R_{1}$ is any quotient ring of $k\left[\left[X_{1}, \cdots, X_{d-1}\right]\right]$ with its maximal ideal $\mathfrak{m}_{1}$ satisfying $\mathfrak{m}_{1}=\mathfrak{m}_{1}^{2}$ (i.e. $R_{1}$ has zero cotangent space), then $R_{1}=k$. In fact, $R_{1}$ is noetherian and we have $\mathfrak{m}_{1}^{l}=\mathfrak{m}_{1}$ for all $l \in \mathbb{Z}_{\geq 1}$, so by Krull's intersection theorem we conclude that $\mathfrak{m}_{1}=0$ and $R_{1}=k$.

Assume $c=1$. Then $\hat{\mathcal{S}} / \overline{\mathfrak{m}}^{p} \cong k$, so $\hat{\mathcal{S}}$ has zero cotangent space and thus $\hat{\mathcal{S}}=k$. Next we treat the case $c \geq 2$. Let $\alpha$ be the composite

$$
\alpha: k\left[\left[X_{1}, \cdots, X_{d-1}\right]\right] \rightarrow \hat{\mathcal{S}} / \overline{\mathfrak{m}}^{p} \stackrel{\beta}{\rightarrow} k[X] / X^{c} .
$$

Let $J=\operatorname{ker} \alpha$. It suffices to prove that $I=J$. Note that because $\beta$ is an isomorphism we have

$$
I+\mathfrak{m}^{p}=J
$$

In the following we prove $\mathfrak{m}^{p} \subset I$, which will imply $I=J$ and hence the theorem. The argument is a variant of [RTZ13, Lemma 11.1].

Let $Y \in k\left[\left[X_{1}, \cdots, X_{d-1}\right]\right]$ be such that $\alpha(Y)=X$. Since $X$ generates the maximal ideal in $k[X] / X^{c}$, we have

$$
\mathfrak{m}=J+(Y) .
$$

Then by (5.4.1.1) and (5.4.1.2) we have $\mathfrak{m}=I+(Y)+\mathfrak{m}^{p}$, and so the local ring $k\left[\left[X_{1}, \cdots, X_{d-1}\right]\right] /(I+(Y))$ has zero cotangent space. We have observed that the cotangent space being zero implies that the ring has to be $k$, or equivalently

$$
\mathfrak{m}=I+(Y)
$$

Now we start to show $\mathfrak{m}^{p} \subset I$. By (5.4.1.3) we have $\mathfrak{m}^{p} \subset I+\left(Y^{p}\right)$, so we only need to prove $Y^{p} \in I$. We will show the stronger statement that $Y^{c} \in I$. By Krull's intersection theorem, it suffices to show that $Y^{c} \in I+\mathfrak{m}^{p l}$ for all $l \geq 1$. In the rest we show this by induction on $l$.

Assume $l=1$. Note that $\alpha\left(Y^{c}\right)=0$, so by (5.4.1.1) we have

$$
Y^{c} \in J=I+\mathfrak{m}^{p} .
$$

Suppose $Y^{c} \in I+\mathfrak{m}^{p l}$ for an integer $l \geq 1$. Write

$$
Y^{c}=i+m, i \in I, m \in \mathfrak{m}^{p l} .
$$

\footnotetext{
${ }^{5}$ We use this notation because previously we used the notation $\mathcal{S}$ to denote the local ring of $S_{\Lambda}^{\bar{g}}$ at $x_{0}$.
} 
By (5.4.1.2) we know

$$
\mathfrak{m}^{p l} \subset(J+(Y))^{p l} \subset \sum_{s=0}^{p l} J^{s}(Y)^{p l-s} .
$$

Thus we can decompose $m \in \mathfrak{m}^{p l}$ into a sum

$$
m=\sum_{s=0}^{p l} j_{s} Y^{p l-s}, j_{s} \in J^{s} .
$$

By (5.4.1.4) and (5.4.1.5), we have

$$
Y^{c}=i+\sum_{s=0}^{p l} j_{s} Y^{p l-s} .
$$

Splitting the summation $\sum_{s=0}^{p l}$ into two sums $\sum_{s=0}^{p l-c}$ and $\sum_{s=p l-c+1}^{p l}$ and moving the sum $\sum_{s=0}^{p l-c}$ to the left hand side, we obtain

$$
Y^{c}-\sum_{s=0}^{p l-c} j_{s} Y^{p l-s}=i+\sum_{s=p l-c+1}^{p l} j_{s} Y^{p l-s}
$$

Denote

$$
A:=\sum_{s=0}^{p l-c} j_{s} Y^{p l-s-c} .
$$

Then the left hand side of (5.4.1.6) is equal to $(1-A) Y^{c}$. Hence we have

$(1-A) Y^{c}=i+\sum_{s=p l-c+1}^{p l} j_{s} Y^{p l-s} \subset I+J^{p l-c+1} \stackrel{\underline{5.4 .1 .1}}{=} I+\left(I+\mathfrak{m}^{p}\right)^{p l-c+1}=I+\mathfrak{m}^{p(p l-c+1)} \subset I+\mathfrak{m}^{p(l+1)}$, where for the last inclusion we have used $c<p$. Since $1-A$ is a unit in $k\left[\left[X_{1}, \cdots, X_{d-1}\right]\right]$ (because $c<p$ ), we have $Y^{c} \in I+\mathfrak{m}^{p(l+1)}$. By induction, $Y^{c} \in I+\mathfrak{m}^{p l}$ for all $l \in \mathbb{Z}_{\geq 1}$, as desired.

Corollary 5.4.2. Let $g \in J_{b}\left(\mathbb{Q}_{p}\right)$ be regular semisimple and minuscule. Assume $\mathrm{RZ}^{g} \neq \varnothing$ and keep the notation of 5.1.3. Let $x_{0} \in\left(\delta\left(\mathrm{RZ}^{b}\right) \cap \mathrm{RZ}^{g}\right)(k)$. Let $\left(\mathcal{L}_{d-1}, \mathcal{L}_{d}\right) \in S_{\Lambda}(k)$ correspond to $x_{0}$ via Proposition 5.1.4 and define $\lambda, c$ as in Definition 5.2.7. Assume $p>c$. Then the complete local ring of $\delta\left(\mathrm{RZ}^{\mathrm{b}}\right) \cap \mathrm{RZ}^{g}$ at $x_{0}$ is isomorphic to $k[X] / X^{c}$. Moreover, we have $c=\frac{m(Q(T))+1}{2}$, where $Q(T)$ as in Theorem 3.6.4. In particular, $1 \leq c \leq n / 2$.

Proof. The first part follows immediately from Proposition 5.1.4 and Theorem 5.4.1. It remains to show that

$$
c=\frac{m(Q(T))+1}{2}
$$

Suppose $x_{0} \in \mathrm{BT}_{\Lambda^{\prime}}$ for some vertex lattice $\Lambda^{\prime}$ (not necessarily equal to $\Lambda=L(g)^{\vee}$ ). Let $L$ be the associated special lattice. Then we have $(2.12)$

$$
\left(\Lambda^{\prime}\right)_{W}^{\vee} \subseteq L \subseteq \Lambda_{W}^{\prime}, \quad\left(\Lambda^{\prime}\right)_{W}^{\vee} \subseteq \Phi(L) \subseteq \Lambda_{W}^{\prime} .
$$

Hence the eigenvalue $\lambda$ of $\bar{g}$ on $\Phi\left(\mathcal{L}_{d}\right) / \mathcal{L}_{d-1} \cong(L+\Phi(L)) / L$ appears among the eigenvalues of $\bar{g}$ on $\Lambda^{\prime} /\left(\Lambda^{\prime}\right)^{\vee}$, and so the minimal polynomial of $\bar{g}$ on $\Lambda^{\prime} /\left(\Lambda^{\prime}\right)^{\vee}$ in $\mathbb{F}_{p}[T]$ is equal to $Q(T)$ by the proof of Theorem 3.6.4. Notice that the characteristic polynomial of $\bar{g}$ on $\Phi\left(\mathcal{L}_{d}\right)$ (in $k[T]$ ) divides $R(T) Q(T)$ (the characteristic polynomial of $\bar{g}$ on $\Lambda_{W}^{\prime} / L(g)$ ) and also is divided by $R(T)$ (the characteristic polynomial of $\bar{g}$ on $\left(\Lambda^{\prime}\right)_{W}^{\vee} / L(g)$ ). It follows that $c$, the multiplicity of $\lambda$ of $\bar{g}$ on $\Phi\left(\mathcal{L}_{d}\right)$, is equal to the multiplicity of $\lambda$ in $R(T) Q(T)$. The desired formula for $c$ then follows since

$$
m(Q(T))+1=2 \text {. the multiplicity of } Q(T) \text { in } R(T) Q(T) .
$$


Finally, we note that $m(Q(T))$ is a positive odd integer not greater than the degree of $P(T)$, and the latter, being the type of the vertex lattice $\Lambda=L(g)^{\vee}$, is an even integer $\leq t_{\max }$ (cf. 2.7 ). The bound for $c$ follows from the value of $t_{\max }$ given in $\$ 2.7$.

\section{REFERENCES}

[BP17] O. Bueltel and G. Pappas, (G, $\mu$ )-displays and Rapoport-Zink spaces, ArXiv e-prints (February 2017), 1702.00291.

[DL76] P. Deligne and G. Lusztig, Representations of reductive groups over finite fields, Ann. of Math. (2) 103(1), 103-161 (1976).

[GGP12] W. T. Gan, B. H. Gross and D. Prasad, Symplectic local root numbers, central critical $L$ values, and restriction problems in the representation theory of classical groups, Astérisque (346), 1-109 (2012), Sur les conjectures de Gross et Prasad. I.

[GK92] B. H. Gross and S. S. Kudla, Heights and the central critical values of triple product L-functions, Compositio Math. 81(2), 143-209 (1992).

[GS95] B. H. Gross and C. Schoen, The modified diagonal cycle on the triple product of a pointed curve, Ann. Inst. Fourier (Grenoble) 45(3), 649-679 (1995).

[GZ86] B. H. Gross and D. B. Zagier, Heegner points and derivatives of L-series, Invent. Math. 84(2), 225-320 (1986).

[Har95] J. Harris, Algebraic geometry, volume 133 of Graduate Texts in Mathematics, Springer-Verlag, New York, 1995, A first course, Corrected reprint of the 1992 original.

[HP14] B. Howard and G. Pappas, On the supersingular locus of the GU(2,2) Shimura variety, Algebra Number Theory 8(7), 1659-1699 (2014).

[HP15] B. Howard and G. Pappas, Rapoport-Zink spaces for spinor groups, ArXiv e-prints (September 2015), 1509.03914.

[Ive72] B. Iversen, A fixed point formula for action of tori on algebraic varieties, Invent. Math. 16, 229-236 (1972).

[Kim13] W. Kim, Rapoport-Zink spaces of Hodge type, ArXiv e-prints (August 2013), 1308.5537.

[Kis10] M. Kisin, Integral models for Shimura varieties of abelian type, J. Amer. Math. Soc. 23(4), 967-1012 (2010).

[Kri16] R. M. Krishna, Relative Trace Formula for SO2 x SO3 and the Waldspurger Formula, ProQuest LLC, Ann Arbor, MI, 2016, Thesis (Ph.D.)-Columbia University.

[Lus11] G. Lusztig, From conjugacy classes in the Weyl group to unipotent classes, Represent. Theory 15, 494-530 (2011).

[Lus77] G. Lusztig, Coxeter orbits and eigenspaces of Frobenius, Invent. Math. 38(2), 101-159 (1976/77).

[MP16] K. Madapusi Pera, Integral canonical models for spin Shimura varieties, Compos. Math. 152(4), $769-824$ (2016).

[RTZ13] M. Rapoport, U. Terstiege and W. Zhang, On the arithmetic fundamental lemma in the minuscule case, Compos. Math. 149(10), 1631-1666 (2013).

[RZ96] M. Rapoport and T. Zink, Period spaces for p-divisible groups, volume 141 of Annals of Mathematics Studies, Princeton University Press, Princeton, NJ, 1996.

[YZZ] X. Yuan, S.-W. Zhang and W. Zhang, Triple product L-series and Gross-Kudla-Schoen cycles, preprint .

[YZZ13] X. Yuan, S.-W. Zhang and W. Zhang, The Gross-Zagier formula on Shimura curves, volume 184 of Annals of Mathematics Studies, Princeton University Press, Princeton, NJ, 2013.

[Zha12] W. Zhang, On arithmetic fundamental lemmas, Invent. Math. 188(1), 197-252 (2012).

E-mail address: chaoli@math.columbia.edu

Department of Mathematics, Columbia University, 2990 Broadway, New York, Ny 10027

E-mail address: yihang@math.harvard.edu

Department of Mathematics, Harvard University, 1 Oxford Street, Cambridge, Ma 02138 